\title{
Minocycline suppresses disease-associated microglia (DAM) in a model of photoreceptor cell degeneration
}

\section{Sarah Doyle ( sarah.doyle@tcd.ie )}

Trinity College Dublin https://orcid.org/0000-0002-6294-9380

\section{Ema Ozaki}

Trinity College Dublin: The University of Dublin Trinity College

Conor Delaney

Trinity College Dublin: The University of Dublin Trinity College

Matthew Campbell

Trinity College Dublin: The University of Dublin Trinity College

\section{Research}

Keywords: disease-associated microglia, minocycline, retinal degeneration, lipoprotein lipase

Posted Date: July 20th, 2021

DOI: https://doi.org/10.21203/rs.3.rs-656962/v1

License: (c) (i) This work is licensed under a Creative Commons Attribution 4.0 International License. Read Full License 
1 Title: Minocycline suppresses disease-associated microglia (DAM) in a model of photoreceptor cell degeneration.

4 Authors: Ema Ozaki ${ }^{1,2}$, Conor Delaney ${ }^{3}$, Matthew Campbell ${ }^{3}$, Sarah L. Doyle ${ }^{1,2,4}$

7 Affiliations:

$8{ }^{1}$ Department of Clinical Medicine, School of Medicine, Trinity College Dublin, Dublin, Ireland.

$9 \quad{ }^{2}$ Trinity College Institute of Neuroscience, Trinity College Dublin, Dublin, Ireland.

$10 \quad{ }^{3}$ Smurfit Institute of Genetics, Trinity College Dublin, Dublin, Ireland.

$11{ }^{4}$ National Children's Research Centre, Our Lady's Children's Hospital Crumlin, Dublin, Ireland.

13 Corresponding Author:

14 Dr. Sarah Doyle, e-mail: sarah.doyle@tcd.ie tel: +35318963011

15

16 Key words: disease-associated microglia, minocycline, retinal degeneration, lipoprotein lipase 


\section{Abstract:}

25 Background: As the resident immune cells in the retina, microglia play important homeostatic 26 roles in retinal immune regulation and neuroprotection. However, chronic microglia activation is 27 a common hallmark of many degenerative retinal diseases. The semi-synthetic broad spectrum 28 tetracycline antibiotic, minocycline appears to inhibit pro-inflammatory microglia which 29 coincides with protection against photoreceptor cell degeneration. A sub-type of microglia 30 termed disease associated microglia (DAM) have recently been associated with a wide range of 31 central nervous system (CNS) diseases.

32 Methods: In this study we examine the kinetics of microglia infiltration towards the outer retina 33 of rhodopsin knockout mice $\left(r h o^{-/-}\right)$by immunofluorescence, and undertake transcriptional and 34 spatial localization analysis of markers for evidence of both homeostatic function and appearance 35 of DAMs.

36 Results: We demonstrate in the Rho-/-mice that $\mathrm{Iba}^{+}$and $\mathrm{P} 2 \mathrm{RY} 12^{+}$microglia take on activated 37 morphology early in disease, prior to notable photoreceptor loss and are capable of infiltrating 38 the subretinal space. Expression of lipid processing enzyme and DAM-marker lipoprotein lipase 39 (LPL) is primarily observed only after microglia have traversed the ONL. Administration of 40 minocycline to Rho-/-mice induced loss of phagocytic/DAM microglia in the outer retina in vivo 41 coinciding with photoreceptor survival and amelioration of retinal degeneration.

42 Conclusion: Our study identifies microglial lipid processing enzyme and DAM-marker LPL as a

43 direct target of minocycline and indicates that suppression of lipid metabolism is one mechanism 44 by which minocycline protects against inflammation induced photoreceptor cell death. 
Introduction

49 Retinal degeneration is a progressive neurodegenerative condition and encompasses a cohort of 50 inherited, acquired and age-related diseases that can lead to severe visual impairment and 51 eventual blindness. Retinitis pigmentosa (RP) is a genetically heterogeneous category of 52 inherited photoreceptor degenerations for which retinal gene therapies targeting X-linked RP 53 with variants in the RPGR gene and RPE65-related Leber congenital amaurosis (LCA) are 54 proving promising. However, while gene therapies may have benefit for those individuals with mutations in the targeted genes, no broad-spectrum effective treatments exist for this condition.

56 Photoreceptor cells are specialised neurons that convert light into electrical signals that are 57 deciphered in the brain. Identification of unifying processes that can protect photoreceptors from 58 premature death has potential to offer a global therapeutic approach for prolonging sight across 59 multiple inherited blinding diseases.

60 Retinal inflammation is thought to accelerate the pace of progression of photoreceptor cell 61 death irrespective of the underlying cause of disease, and suppression of microglial activation 62 has been shown to be neuroprotective in mouse models of retinal degeneration (Peng, Xiao et al. 63 2014, Murakami, Ishikawa et al. 2020). Recent advances in single-cell analysis have uncovered 64 subtleties in microglia subtypes that are associated with a wide range of neurodegenerative 65 diseases termed DAM (disease associated microglia) (Keren-Shaul, Spinrad et al. 2017). These 66 DAM appear to be highly effective at phagocytosing debris and unwanted protein aggregates and 67 may in fact be beneficial if triggered early in disease where strong phagocytic activity is 68 desirable. 
69 In this study, we examine the kinetics of microglia infiltration towards the outer retina of 70 rhodopsin knockout mice $\left(R h o^{-/}\right)$, and undertake transcriptional and spatial localization analysis 71 of markers for evidence of both homeostatic function and appearance of DAM. The Rho-/-retina 72 develops normal numbers of rod and cone nuclei, but the rods have no outer segments and 73 ensuing rod degeneration becomes evident histopathologically from 3 weeks (Ozaki, Gibbons et 74 al. 2020). Rod photoreceptor degeneration is followed by cone photoreceptor degeneration with 75 a complete loss of electrical responsiveness by $8 \mathrm{wk}$. At $12 \mathrm{wk}$, the majority of photoreceptors in 76 the retina are lost (Humphries, Rancourt et al. 1997). In this study, we demonstrate in the Rho-/77 mice that $\mathrm{Iba1}^{+}$and $\mathrm{P} 2 \mathrm{RY} 12^{+}$microglia take on activated morphology early in disease, prior to notable photoreceptor loss and are capable of infiltrating the subretinal space (SRS), an immune 79 privileged site usually devoid of microglia. Prior to crossing through the photoreceptor outer nuclear layer (ONL) microglia express CD68 further indicating presence of an inflammatory

81 environment. Interestingly expression of lipid processing enzyme and DAM-marker lipoprotein 82 lipase (LPL) is primarily observed only after microglia have traversed the ONL. Recent literature 83 indicates that the semi-synthetic broad spectrum tetracycline antibiotic, minocycline, may have 84 potential to counter-regulate pro-inflammatory microglia and protect photoreceptor cell 85 degeneration (Peng, Xiao et al. 2014, Scholz, Sobotka et al. 2015, Terauchi, Kohno et al. 2021). 86 Administration of minocycline to Rho-/-mice reduced markers of DAM in the degenerating Rho87 /-retina and prevented microglia infiltration into the SRS. Minocycline has many cell and 88 molecular targets; here we show that it acts directly on microglial cells, significantly reducing 89 DAM-markers APOE and LPL among others, in vitro, and furthermore, inhibits microglia 90 phagocytic function. In turn, minocycline-induced loss of phagocytic/DAM microglia in the 91 outer retina in vivo coincides with photoreceptor survival and amelioration of retinal 
92 degeneration. Our study identifies microglial LPL as a direct target of minocycline and indicates

93 that suppression of lipid metabolism is one mechanism by which minocycline protects against

94 inflammation induced photoreceptor cell death.

95

96 Materials and Methods

97 Animals

98 All studies carried out in the Smurfit Institute of Genetics in TCD adhere to the principles laid 99 out by the internal ethics committee at $\mathrm{TCD}$, and all relevant national licenses were obtained 100 before commencement of all studies. C57BL/6J mice were sourced from Jackson Laboratory and

101 bred on-site at the Smurfit Institute of Genetics in TCD. Rho-/-mice were kindly provided by 102 Prof Peter Humphries, TCD. Before experiments, all mice were kept on a 12-h light/dark cycle.

104 Minocycline administration

105 Rho-/-mice received intra-peritoneal injections of $50 \mathrm{mg} / \mathrm{kg}$ minocycline (Sigma-Aldrich) or 106 vehicle $\mathrm{H}_{2} \mathrm{O}$ twice daily, starting from postnatal day 16 and continuing till postnatal day 21 or 10742.

108

109 Optical Coherence Tomography (OCT) analysis

110 OCT was performed on mice using a Heidelberg Spectralis OCT (Heidelberg Engineering).

111 Pupils were dilated with $1 \%$ tropicamide and $2.5 \%$ phenylephrine and mice anaesthetized using 112 a mixture of ketamine $/$ medetomidine $(100 / 0.25 \mathrm{mg} / \mathrm{kg})$. OCT images were captured with a $30^{\circ}$ 113 angle of view. Heidelberg eye explorer version 1.7.1.0 was used to capture 
114 images. ImageJ (National Institutes of Health, Rockville, MD, USA) was used for quantification 115 analysis.

\section{$117 \quad$ H\&E staining}

118 Mouse eyes were fixed in Davidson's Fixative for $24 \mathrm{~h}$, followed by three PBS washes. Eyes 119 were processed in a tissue processor under gentle agitation as follows: $70 \%$ ethanol for $1 \mathrm{~h}, 80$ $120 \%$ ethanol for $1 \mathrm{~h}, 95 \%$ ethanol for $1 \mathrm{~h}, 100 \%$ ethanol for $1 \mathrm{~h}, 100 \%$ ethanol for $1 \mathrm{~h}, 50 \%$

121 ethanol/xylene mix for $1 \mathrm{~h}$, xylene for $1 \mathrm{~h}$, xylene for $1 \mathrm{~h}$, paraffin at $60^{\circ} \mathrm{C}$ for $1 \mathrm{~h}$, and paraffin 122 under vacuum at $60{ }^{\circ} \mathrm{C}$ for $1 \mathrm{~h}$. Eyes were then embedded in paraffin and $5 \mu \mathrm{m}$ sections were 123 collected onto Polysine slides using a microtome. The sections were deparaffinized by dipping 124 ten times in xylene, followed by rehydration in 10 dips each of $100 \%, 90 \%$, and $70 \%$ ethanol. 125 The slides were incubated in haemotoxylin solution for $6 \mathrm{~min}$, rinsed in water, and then 126 incubated in eosin solution for 2 min. The slides were rinsed in water and dehydrated by dipping 127 ten times in $70 \%, 90 \%$, and $100 \%$ ethanol and once in xylene. The slides were mounted using 128 the Sub-X Mounting Medium and analyzed under a light microscope (Olympus 1X81).

\section{Immunohistochemistry}

131 For retinal cryosections, mouse eyes were fixed in $4 \%$ paraformaldehyde for $1 \mathrm{~h}$ and $30 \mathrm{~min}$ at 132 room temperature, followed by three PBS washes. Eyes were cryoprotected in $20 \%$ sucrose for $1331 \mathrm{~h}$, followed by $30 \%$ sucrose overnight at $4{ }^{\circ} \mathrm{C}$, and subsequently embedded and frozen in an 134 optimum cutting temperature compound. 12- $\mu \mathrm{m}$ sections were collected onto Polysine slides 135 using a cryostat. Cryosections were block and permeabilized with 5\% NGS and 0.05\% Triton in 136 PBS for $1 \mathrm{~h}$ at room temperature. The slides were incubated overnight at $4^{\circ} \mathrm{C}$ in a humidity 
137 chamber with primary antibody diluted in $5 \%$ NGS. Primary antibodies used were Iba1 (Wako, 138 019-19741, 1:500), CD68 (Abcam, ab53444, 1:200), P2RY12 (AnaSpec ANA55043A, 1:100), 139 and LPL (Abcam, ab21356, 1:100). After three PBS washes, the cryosections were incubated 140 with Alexa Fluor 594- and 488-conjugated goat anti-rabbit, Alexa Fluor 488-conjugated 141 anti-mouse and Alexa Fluor 594-conjugated anti-rat secondary antibodies (1:500; Invitrogen) 142 diluted in 5\% NGS for $2 \mathrm{~h}$ at room temperature and counterstained with Hoechst 33342 143 (1:10,000). The slides were mounted with Hydromount (VWR) mounting medium and analyzed 144 using a confocal microscope (Zeiss LSM 710).

146 For retinal flat-mounts, mouse eyes were fixed in $4 \%$ paraformaldehyde for $15 \mathrm{~min}$. After a PBS 147 wash, the cornea and lens were carefully removed, and four incisions were made into the retinal 148 eye cup to flatten out the tissue. Retinal flatmounts were fixed for a further $15 \mathrm{~min}$ in $4 \%$ 149 paraformaldehyde. After three PBS washes, flat-mounts were permeabilized and blocked 150 overnight in $10 \%$ NGS and $1 \%$ Triton in PBS at $4^{\circ} \mathrm{C}$. Flatmounts were incubated with Iba1

151 antibody for $48 \mathrm{~h}$ at $4^{\circ} \mathrm{C}$ in a humidity chamber. After three PBS washes flat-mounts were 152 incubated with Alexa Fluor 594-conjugated anti-rabbit secondary antibody for $24 \mathrm{~h}$ at $4{ }^{\circ} \mathrm{C}$. Flat153 mounts were mounted with Hydromount (VWR) mounting medium and analyzed using a 154 confocal microscope (Zeiss LSM 710) and ImageJ software. All image analysis was performed 155 using ImageJ.

\section{Cell culture}

158 The mouse microglial BV2 cell line was cultured in RPMI with $10 \%$ FBS, supplemented with 10 $159 \%$ fetal calf serum (Sigma-Aldrich) and $1 \%$ penicillin/streptomycin (Sigma-Aldrich) and 
160 maintained at $37{ }^{\circ} \mathrm{C}$ in a humidified $5 \% \mathrm{CO}_{2}$ atmosphere. The cells were seeded at $5 \times 10^{4}$ cells

161 per $\mathrm{cm}^{2}$ and incubated overnight before treatment. BV2 cells were treated $5 \mathrm{mg} / \mathrm{ml}$ minocycline

162 or sterile $\mathrm{H}_{2} \mathrm{O}$ as vehicle control. Cells were harvested $6 \mathrm{~h}$ and $24 \mathrm{~h}$ after treatment.

163

164 Phagocytosis Assay

165 BV2 cells were plated on chamber slides and treated with $5 \mathrm{ug} / \mathrm{ml}$ minocycline for $21 \mathrm{~h}$ prior to

166 being assayed. Polystyrene amine modified (yellow-green) latex beads (Sigma-Aldrich) were

167 pre-opsonised in FBS $(1: 5)$ at $37{ }^{\circ} \mathrm{C}$ for $1 \mathrm{~h}$. Opsonized beads were diluted 1:2,000 in media and 168 added to cells for $3 \mathrm{~h}$. Cells were washed with ice-cold PBS 3 times to remove surface bound

169 beads, fixed in 4 \% PFA for 10 min and stained with MitoTracker Orange CMTMRos for 17030 min. Cells were washed and counterstained with Hoechst 33342 (1:10,000). Cells were 171 imaged on a BX51 Olympus microscope and analyzed by ImageJ.

\section{3 qRT-PCR analysis}

174 Total RNA was extracted from BV2 cells or mouse retinas using Isolate II RNA extraction kit 175 (Bioline) as per the manufacturer's instructions. RNA was reverse transcribed using MMLV 176 Reverse Transcriptase (Promega). Target genes were amplified by real-time PCR with SensiFast 177 SYBR Green (Bioline) using the ABI 7900HT system (Applied Biosystems). The comparative 178 CT method was used for relative quantification after normalisation to the "housekeeping" gene 179 ubiquitin C (UBC). Primers used were as follows: 


\begin{tabular}{|l|l|l|}
\hline Target & Forward Primer (5'-3') & Reverse Primer (5'-3') \\
\hline APOE & ATTGCTGACAGGATGCCTAGC & GGTTGGTTGCTTTGCCACTC \\
\hline AXL & TTCAACTGTGCTACGTCCCC & GGGTCCCTCTAGGTAAGCCA \\
\hline CLEC7a & GTGGTAGTAGTGGTTGCTGC & ATTCTGTGGGCTTGTGGTTC \\
\hline CSF1R & AAGCAGAAGCCGAAGTACCA & GTCCCTGCGCACATATTTCAT \\
\hline CTSB & CAGGCTGGACGCAACTTCTA & GCCCCAAATGCCCAACAAG \\
\hline CX3CR1 & CTGTTATTTGGGCGACATTG & AACAGATTTCCCACCAGACC \\
\hline iNOS & ATGGACCAGTATAAGGCAAGC & GCTCTGGATGAGCCTATATTG \\
\hline ITGAX & CGATGCCTTCCCATGAATACG & CTTGGTGTCTCTGTGCCCTC \\
\hline LGALS3 & CTCTGACAGCTAGCGGAGC & AGGCATCGTTAAGCGAAAAGC \\
\hline LPL & TCGTCATCGAGAGGATCCGA & TGTTTGTCCAGTGTCAGCCA \\
\hline P2RY12 & CAAGGGGTGGCATCTACCTG & AGGCAGCCTTGAGTGTTTCTG \\
\hline SALL1 & TTTCCAATCCGACCCCGAAG & CCACAGACATGGGCATCCTT \\
\hline SELPLG & GGGATGGTCCTTCCTTTGGG & ACAATGGTCTAAGCGCCCTC \\
\hline SPP1 & CTGGCTGAATTCTGAGGGACT & CTGCTTCTGAGATGGGTCAGG \\
\hline TGFBR1 & AGCTCCTCATCGTGTTGGTG & GGCCTGTCTCGAGGAATTAGG \\
\hline TMEM119 & TTCACCCAGAGCTGGTTCCATA & TCTCCGGTGTGGGACTGAA \\
\hline TREM2 & ACAGCACCTCCAGGAATCAAG & AGGATCTGAAGTTGGTGCCC \\
\hline UBC & GGTGTACTGGCTGGGATTGT & GCAATGTGTTGTTTCCGGGT \\
\hline
\end{tabular}

183 


\section{Western blot analysis}

187 Retinal tissue and BV2 cells were lysed in RIPA lysis buffer with phosphatase and protease 188 inhibitors (Sigma-Aldrich) and centrifuged at 15,000 $\mathrm{g}$ for $15 \mathrm{~min}$. Protein lysates were resolved 189 on $10 \%$ or $12 \%$ SDS polyacrylamide gels and transferred to a PVDF membrane. Membranes 190 were blocked in $5 \%$ non-fat milk in Tris-buffered saline containing $0.05 \%$ Tween-20 (TBST) 191 for $1 \mathrm{~h}$ and then incubated overnight at $4{ }^{\circ} \mathrm{C}$ in primary antibodies against APOE (Merck, 192 178479, 1:2000), LPL (Abcam, ab21356, 1:1000), Galectin-3 (R\&D, AF1197, 1:2000), CSF1R 193 (Invitrogen, PA5-25974, 1:500) and $\beta$-actin (1:2,000; Sigma-Aldrich). After three TBST washes, 194 the membranes were incubated in horseradish peroxidase-conjugated anti-rabbit, anti-mouse or 195 anti-goat antibodies (1:2,000; Sigma-Aldrich) for $1 \mathrm{~h}$ at room temperature. After three TBST 196 washes, the membranes were developed using enhanced chemiluminescence (Advansta).

197 Densitometry was performed using ImageJ, with SARM1 levels normalized to the loading 198 control $\beta$-actin.

199

200

201

202

203

204

205

206

207

208 


\section{Results}

211 Microglia are activated and infiltrate the outer retina in the rhodopsin knockout model of 212 photoreceptor degeneration

214 Microglia are morphologically and functionally dynamic cells that can transform from a highly 215 ramified state into a rod or amoeboid state during activation and pathology. Under basal or 216 physiological conditions, ramified microglia predominate. However, in response to injury or 217 inflammatory conditions, de-ramification occurs where microglial processes shorten and thicken 218 and cell bodies expand, ultimately shifting into a round amoeboid shape (Karperien, Ahammer et 219 al. 2013). Here, we examine microglia dynamics in the Rho-/-model of retinal degeneration. 220 Rho-/-mice develop a normal number of rod and cone nuclei but with the absence of rod outer 221 segments (OS), these cells begin to degenerate from 3 weeks of age and by 12 weeks most of the 222 photoreceptors are lost. To visualize and characterize the role of microglia in the Rho-/-model of 223 retinal degeneration we performed immunofluorescence on retinal flatmounts of the microglia 224 marker Iba1 in wild-type and Rho-/-mice at 3, 6, 9 and 12 weeks of age (Fig. 1A). At all time225 points observed, wild-type microglia had a ramified appearance with long fine processes and 226 small cell bodies indicative of physiological 'surveillant' microglia (Fig. 1A, first column). In 227 the contrary, microglia in the Rho-/-retinal flatmounts had an activated appearance with larger 228 cell bodies and retracted processes (Figure 1A, second column), which are particularly evident 229 in the high magnification and skeleton images (Fig. 1A, a'-h'). De-ramified microglia were 230 evident from as early as 3 weeks of age (Fig. 1A, a', b'), demonstrating that microglial 231 activation occurs from a very early age in the Rho-/-mice. To examine the distribution of 
232 microglia within the laminar structure of the retina in the Rho-/-mice, we stained cross sections

233 of the retina from these mice at 3, 6, 9 and 12 weeks of age with Iba1. In the wild-type retina,

234 Iba1-stained microglia were observed in the synaptic inner plexiform (IPL) and outer plexiform

235 layers (OPL) (Fig. 1B, first column). However, in the retinal cryosections from Rho-/-mice,

236 Iba1-stained microglia were seen to migrate into the outer nuclear layer (ONL) and down

237 towards the photoreceptor inner and outer segments or sub-retinal space (SRS) (Figure 1B,

238 second column).

240 Microglia infiltrating the SRS are actively phagocytosing photoreceptor cells and express 241 disease associated microglia (DAM) marker LPL

242 Next, we sought to analyse the microglia infiltrating the SRS for markers of phagocytosis and 243 markers of homeostatic function. First, we stained retinal cryosections from 12 week old wild244 type mice (Fig. 2A, top row) and Rho-/-mice at 3, 6, 9 and 12 weeks of age with CD68, a 245 lysosomal protein that is upregulated in actively phagocytic cells. In wild-type sections, no co246 localization of CD68 and Ibal were observed, and fluorescence was mainly seen in the OPL

247 likely due to non-specific IgG staining in the blood vessels (Fig. 2A, top row). CD68 positive 248 staining was significantly increased in Rho-/-cryosections and co-localization of Iba1 and CD68 249 was observed in microglia in the OPL and in microglia that had infiltrated the ONL and SRS 250 (Fig.s 2A a' \& $\mathbf{2 A} \mathbf{b}^{\prime}$ ). As Ibal is also expressed in macrophages, we next stained retinal 251 cryosections with the more recently identified microglial-specific homeostatic marker P2RY12 252 (Fig. 2B). Although some studies have found a decrease in microglial homeostatic genes like 253 P2RY12 in aging and neurodegenerative models, we found P2RY12 immunopositivity in both 254 wild-type cryosections (Fig. 2B, top row) and Rho-/-cryosections at 3, 6, 9 and 12 weeks of age 
255 (Fig. 2B, rows 2-5). Co-staining with CD68 also revealed co-localization of CD68 and P2RY12 256 in the activated microglia in the OPL, ONL and SRS of Rho-/-mice (Fig.s 2B a' and 2B b'). 257 Finally, retinal cryosections from wild-type and Rho-/-mice were stained with lipoprotein lipase 258 (LPL) (Fig. 3). LPL, an enzyme that hydrolyses triglyceride rich lipoproteins, is associated with 259 an alternatively activated and highly phagocytic microglial phenotype. In wild-type retinas, no

260 LPL reactivity was observed in microglia with fluorescence only observed in blood (either due to 261 non-specific IgG staining or specific staining of vessel walls) (Fig. 3A). In contrast, LPL 262 immunopositivity was observed in Rho-/-retinas at 3, 6, 9 and 12 weeks of age and co-staining 263 with Iba1 revealed co-localisation with microglia that resided solely in the SRS; Iba1-positive 264 microglia in the inner retina remained LPL-negative (Fig.s 3A a' b' c' $\&$ d', white arrows).

266 Minocycline downregulates DAM genes in the rhodopsin knockout model of retinal 267 degeneration

268 Minocycline, a broad-spectrum tetracycline antibiotic, has previously been reported to ameliorate 269 photoreceptor cell death in numerous mouse models of retinal degeneration (Zhang, Lei et al. 270 2004, Kohno, Chen et al. 2013, Peng, Xiao et al. 2014, Scholz, Sobotka et al. 2015, Dannhausen, 271 Möhle et al. 2018, Terauchi, Kohno et al. 2021) and is a known inhibitor of microglial activation. 272 Given the interesting LPL staining pattern we had observed in the Rho-/-retina, we wanted to 273 examine the effects of minocycline on genes associated with homeostatic function and those that 274 are a signature of disease-associated microglia (DAM). DAM-related genes of which LPL is a 275 key member have been revealed due to recent advances in single cell technologies. We injected 276 Rho-/-mice with $50 \mathrm{mg} / \mathrm{kg}$ of minocycline or vehicle $\left(\mathrm{H}_{2} \mathrm{O}\right)$ control twice a day from post-natal 277 day 16 (P16) until the mice were sacrificed at 3 weeks of age. Immunofluorescence of Iba1 in 
278 retinal flatmounts from these mice showed a significant reduction in activated microglia with

279 minocycline treatment, with the cells having a more ramified morphology compared to vehicle

280 injected mice (Fig. 4A). The difference in morphology was confirmed using the grid cross

281 method where the ramified microglia from minocycline injected animals had greater numbers of

282 grid cross points compared to the shorter cellular processes of the vehicle injected Rho-/-mice

283 (Fig. 4B). We then assessed the neural retina of minocycline or vehicle injected Rho-/-mice for

284 signature markers of homeostatic microglia and DAM. Out of the 6 homeostatic microglial genes

285 (Cx3crl, P2ry12, Sall1, Tgfbrl, Selplg, Tmem119) assessed by quantitative real-time PCR,

286 expression levels of 5 remained unchanged while Sall1 was found to be downregulated in retinas

287 from minocycline injected mice (Fig. 4C). Interestingly, the DAM signature genes (Apoe, Axl,

288 Clec7a, Ctsb, Csf1r, Itgax, Lgals3, Lpl, Spp1, Trem2, Tyrobp) were found to be more

289 transcriptionally dynamic with a reduction in Apoe, Axl, Clec7a, Csf1r, Ctsb and Lpl levels in

290 retinas from minocycline injected mice while Itgax levels increased (Fig. 4C). The gene

291 expression profiles of these microglial genes are illustrated in a heatmap (Fig. 4D) and also

292 shows the levels of expression of these genes in the retina with genes most highly expressed in

293 the retina in red and those least expressed in blue. As one of the most significantly DAM genes

294 downregulated by minocycline at the transcriptional level, LPL levels were further examined in

295 retinal cryosections from these mice. In vehicle-injected 3-week-old Rho-/-mice, LPL and Iba1

296 co-localization was observed in microglia residing in the SRS whereas LPL was absent in all

297 microglia in minocycline injected animals (Fig. 4E). 
301 Minocycline directly regulates DAM gene expression in BV2 microglia in vitro.

302 As the neural retina is composed of many different cell types, we next sought to examine how 303 minocycline affects homeostatic and DAM gene expression profiles specifically in microglia, 304 using the BV2 mouse microglia cell line. Minocycline is known to inhibit M1 polarization in 305 microglia, (Kobayashi, Imagama et al. 2013) so we first confirmed by quantitative real-time PCR 306 analysis that transcriptional levels of the M1 marker iNOS was significantly reduced in BV2 307 cells after minocycline $(5 \mathrm{ug} / \mathrm{ml})$ treatment $\left(\right.$ Fig.5A $\mathbf{1}^{\text {st }}$ panel). Interestingly, we saw no 308 significant changes in the 6 homeostatic microglial genes (Cx3crl, P2ry12, Sall1, Tgfbrl, Selplg, 309 Tmem 119) assessed while minocycline significantly reduced 3 of the DAM genes, Clec $7 a, L p l$ 310 and Tyrobp (Fig. 5A). Again, the gene expression profiles of these microglial genes are

311 illustrated in a heatmap (Fig. 5B) with the most highly expressed genes in red and the least 312 expressed in blue. Next, we investigated whether minocycline could alter the protein expression 313 levels of some of these microglial markers by Western blot analysis. Similar to what was 314 observed at the transcriptional level, minocycline significantly reduced LPL protein expression 315 (Fig. 5C). As LPL is a key enzyme involved in lipid metabolism, we also examined levels of 316 apolipoprotein E (ApoE), a lipid and cholesterol transporter that is prominently associated with 317 neurodegenerative disease. Although we did not see any changes in Apoe at the transcriptional 318 level in BV2 cells, minocycline significantly reduced both 34-kDa full-length ApoE protein as 319 well as ApoE fragments observed at 28- and 22-kDa (Fig. 5D). CSF1R (colony stimulating 320 factor 1) levels were also significantly decreased by minocycline (Fig. 5E), despite not being 321 altered at the transcriptional level. CSF1R is a primary regulator of microglial, proliferation, 322 migration, differentiation and survival and has been found to be upregulated in inflammatory and 323 neurodegenerative disease. Finally, we looked at protein levels of the DAM marker Galectin-3. 
Lgasl3, the gene encoding galectin-3, was significantly upregulated in the minocycline injected Rho-/-retinas, however, protein levels remained unchanged in minocycline treated BV2 cells

326 (Fig. 5F).

Minocycline inhibits microglial phagocytic function and supports photoreceptor survival in the $R$ ho-/-retinal degeneration model.

330 It was clear that minocycline reduced LPL-positive microglia in the SRS of the Rho-/-retina (Fig.

331 4E). We sought to further examine the effect of minocycline influence on microglia migration.

332 Retinal cryosections from Rho-/-mice that had received two daily injections of minocycline or

333 vehicle control from P16 to P21 or P42, were stained with Iba1 and CD68 to more broadly mark

334 actively phagocytosing cells. Iba1 positive microglial cells were clearly observed in the IPL,

335 OPL, ONL and SRS, in vehicle injected mice, but the number of Iba1 positive cells in the ONL

336 and SRS was significantly reduced in mice treated with minocycline at both 3 weeks (Fig.s 6A

$337 \&$ 6C) and 6 weeks (Fig.s 6B \& 6D) of age, with the majority of microglia residing in the IPL 338 and OPL. Furthermore, co-localization of CD68 and Iba1 was evident in the OPL, ONL and SRS 339 in vehicle injected mice, but this was greatly reduced in minocycline-injected animals. As the 340 majority of cells that were CD68+Iba1+ double-positive were those that had migrated towards

341 the dying photoreceptors, it was difficult to decipher whether minocycline was simply slowing 342 down the migration of these cells towards the SRS or whether minocycline was directly reducing 343 the cells' phagocytic ability. To overcome this we examined whether minocycline could alter the 344 phagocytic capacity of microglia in a basal state in vitro. Untreated and minocycline-treated BV2 345 cells were incubated with green fluorescent latex beads for 3 hours and internalization of the 346 beads was measured by immunofluorescence (Fig. 6E). Indeed, minocycline treatment resulted 
347 in a marked reduction in the number of BV2 cells that had engulfed the latex beads compared to

348 untreated cells (Fig. 6F), indicating that minocycline could directly reduce the basal phagocytic

349 capacity of these cells.

350 Finally, we assessed the therapeutic efficacy of minocycline administration in our Rho-/-model.

351 As before, minocycline was administered by two daily injections from P16 to P42 and we

352 evaluated retinal degeneration in vivo by optical coherence tomography (OCT). OCT uses infra-

353 red light waves to take cross section images of the retina and is a non-invasive mode of

354 measuring retinal thickness and abnormalities in vivo. We measured the distance between the

355 OPL (which forms the interface between the bipolar cells and photoreceptors) and the RPE to get

356 the thickness of both the ONL and the photoreceptor segments and found a substantial

357 preservation in retinal thickness in minocycline-injected Rho-/-mice (Fig. 6G). We also

358 examined photoreceptor cell death by H\&E staining of paraffin-embedded retinal tissue sections

359 from these mice. We counted the number of nuclei rows in the ONL of each eye at 12 points

360 along the vertical meridian from sections cut on a sagittal plane through the optic nerve head at

$361 \sim 150$ and $\sim 250 \mu \mathrm{m}$ from the periphery (Fig. 6I). In support of the OCT data depicting

362 minocycline-induced preservation of retinal thickness, ONL counts indicated minocycline

363 attenuated photoreceptor cell death in the Rho-/-mice with a significant preservation of the

364 numbers of photoreceptor rows still remaining at 6 weeks of age (Fig. 6J). Quantification of the

365 distance from the RPE to the external limiting membrane demonstrated no significant changes

366 with minocycline treatment, indicating that minocycline preserves photoreceptor cell numbers

367 and not the length of the inner segments of the rods, and inner and outer segments of the cones

368 (Fig. 6I). 


\section{Discussion}

371 As the resident immune cells in the retina, microglia play important homeostatic roles in retinal 372 immune regulation and neuroprotection. However, under continuous pathological insult,

373 uncontrolled and chronic microglia activation ensues, and is a common hallmark of many

374 degenerative retinal diseases. Here, we investigate how minocycline, a semi-synthetic

375 tetracycline analogue, affects molecular signatures of homeostatic and disease-associated 376 microglia (DAM) in vivo using the Rho-/-model of retinal degeneration and in vitro using the 377 murine microglia BV2 cell line. Due to the recent surge in single cell RNA transcriptomics, 378 microglia signatures in homeostatic versus diseased phenotypes have been well defined, 379 identifying this DAM subtype. The activation of homeostatic microglia into DAM is a two-step 380 process, with a clear intermediary state observed. In the initial stage, there is a downregulation of 381 microglial homeostatic genes (e.g. P2ry12, Cx3crl, Tmem119, Tgfbrl) along with the 382 upregulation of a subset of DAM genes (e.g. Tryobp, Apoe, B2m and Ctsb). Induction of the key 383 genes involved in lipid metabolism and phagocytosis (e.g. Lpl, Itgax, Clec7a, Trem2) are not 384 observed until the second stage of DAM activation, a stage which is dependent on TREM2 385 signalling (Keren-Shaul, Spinrad et al. 2017).

386 Here, we found that microglia are activated and infiltrate the outer retina in the Rho-/387 model, and this is observed from even 3 weeks of age at the onset of photoreceptor cell death. 388 These microglia infiltrating the SRS are actively phagocytosing and express the DAM marker 389 LPL. Surprisingly, LPL appears only to be induced on encountering the lipid rich environment in 390 the inner and outer segments. Even on traversing through the ONL, we observe no LPL positive 391 stain on the inner retinal side of the external limiting membrane (ELM), despite these microglia 392 being positive for the phagocytic marker CD68; CD68 is apparent in $\mathrm{Iba}^{+}$and P2RY12 cells in 
393 the INL prior to deeper infiltration into the outer retina. Of note, LPL has been shown to co-

394 localize with microglia that have internalized amyloid beta $(\mathrm{A} \beta)$ in mouse and human brains

395 (Keren-Shaul, Spinrad et al. 2017), suggesting that LPL plays a direct role in A $\beta$ uptake and 396 perhaps, in our Rho-/-model, only the microglia that have already phagocytosed the 397 photoreceptor segments in the SRS are LPL positive.

398 Minocycline has been widely used in CNS studies as a microglial inhibitor, however, its 399 effect on the DAM profile has never been investigated. Thus, we next explored whether 400 minocycline could modify DAM signature genes in the retina of Rho-/-mice as well as in BV2 401 microglial cells directly. Interestingly, we found that Sall1 levels showed a decrease with 402 minocycline treatment in Rho-/-animals while the remainder of the homeostatic genes, P2ryl2, $403 \mathrm{Cx} 3 \mathrm{crl}$, Tmem119, Tgfbr1 and Selplg remained unchanged. Sall1 encodes a transcription 404 regulator that dampens a reactive microglia phenotype allowing them to conduct their 405 physiological functions (Buttgereit, Lelios et al. 2016), so it is surprising that this gene was 406 down-regulated with minocycline. However, minocycline did not directly alter this or any other 407 homeostatic genes in microglia in vitro.

408 Remarkably, many genes associated with both the first (Tyrobp, Apoe and Ctsb) and 409 second stage ( $\mathrm{Lpl}$, Csflr and Clec $7 a)$ of DAM activation were down-regulated with minocycline 410 treatment in the retinas from Rho-/-animals and/or BV2 microglia. TYROBP is a cytoplasmic 411 adaptor protein for the immunoreceptor TREM2, and this signalling complex promotes A $\beta$ 412 clearance, enhancing microglial phagocytosis while supressing inflammatory cytokine 413 production and secretion from these cells (Ma, Jiang et al. 2015). Furthermore, triggering of this 414 receptor induces APOE activation, and the APOE-TREM2 signalling pathway has been shown to 415 be a critical regulator of microglia phenotypic change in neurodegenerative disease (Krasemann, 
416 Madore et al. 2017). Intriguingly, a recent study has reported possible APOE-TYROBP

417 signalling independent of TREM2 and propose that this pathway could be an early or initiating 418 step in DAM activation (Audrain, Haure-Mirande et al. 2021). Although, Trem2 levels remained 419 unchanged, both Apoe and Tyrobp levels decreased with minocycline treatment, suggesting that 420 minocycline could reduce TYROBP levels, leading to decreased TREM2 activation and in turn 421 reduced APOE-TREM2 signalling, halting DAM phenotype activation. Additionally, 422 minocycline could also reduce this recently reported APOE-TYROBP signalling directly, 423 dampening the initiation of DAM.

Upregulation of phagocytic and lipid metabolism genes are characteristic of stage two 425 DAM activation suggesting a preference of lipids to fuel the greater bioenergetics needs of these 426 phagocytic microglia (Loving and Bruce 2020). Indeed, knockdown of LPL in BV2 cells has 427 previously been shown to reduce microglial phagocytosis of A $\beta$ (Ma, Bao et al. 2013). Here, we 428 found that minocycline reduced LPL levels in Rho-/-retina and in BV2 microglia both at the 429 transcriptional and protein level. Clec7a, the gene encoding the pattern recognition receptor, 430 Dectin-1, is a known inducer of LC3-associated phagocytosis (Ma, Becker et al. 2012) and 431 although expressed at very low levels in the Rho-/-retina and BV2 microglia, minocycline also 432 significantly reduced Clec $7 a$ expression.

433 Lgals3, the gene encoding Galectin-3, and Itgax (Integrin subunit alpha X), encoding 434 CD11c were two DAM genes surprisingly increased with minocycline in the Rho-/-retinas. 435 Galectin-3 has been found to be highly upregulated in the brains of neurodegenerative disease 436 and was recently identified as a novel endogenous TREM2 ligand (Boza-Serrano, Ruiz et al. 437 2019). Although an increase in Lgals3 was observed transcriptionally in Rho-/-retinas, this 438 galectin is also expressed in other retinal cells such as Muller glia, and indeed, minocycline 
failed to alter both Lgals3 transcriptional and Galectin-3 protein levels in the BV2 microglia cells. Similarly, Itgax which is also expressed in vascular endothelial cells and Muller glia, 441 remained unchanged in minocycline-treated BV2 cells.

As many of the DAM genes minocycline targeted were involved in phagocytosis, we examined the basal phagocytic capacity of microglia in vitro and found that minocycline directly 444 reduced microglial phagocytosis in these cells. Furthermore, we discovered that these 445 minocycline treated microglia, which had a reduced phagocytic capacity and DAM phenotype, 446 could attenuate photoreceptor cell loss in the Rho-/-model of retinal degeneration. Rho-/-mice 447 that were administered with minocycline from P16-42, had delayed retinal thinning than vehicle 448 injected mice when examined in vivo by OCT imaging and from analysis of H\&E stained retinal 449 sections.

Overall, our data supports other studies demonstrating the therapeutic efficacy of 451 minocycline in retinal degeneration; minocycline treatment has previously been shown to 452 preserve the morphology and function of photoreceptors in the $r d 10$ mouse model (Peng, Xiao et 453 al. 2014), in a light-damage-induced RD model (Scholz, Sobotka et al. 2015) and more recently, 454 in the Mertk $^{-}$mouse model of RD (Terauchi, Kohno et al. 2021). It is important to consider, 455 however, that triggering early DAM activation may be useful to induce a microglia response in 456 diseases where a strong phagocytic activity might be beneficial. Indeed, a recent study using 457 single-cell RNA sequencing to characterize the transcriptome of sub-retinal microglia (srMG) in 458 a light damage model of photoreceptor degeneration as well as a genetic model $\left(\mathrm{Rho}^{\mathrm{P} 23 \mathrm{H} / \mathrm{wT}}\right)$ of 459 chronic retinal degeneration (O'Koren, Yu et al. 2019) found that the srMG expressing many 460 DAM markers protected the RPE from disease-associated damage in both models. Additionally, 461 in a model of acute retinal detachment, microglia have been shown to be protective, by rapidly 
462 phagocytosing damaging cell debris to avoid triggering apoptosis of neighbouring photoreceptors

463 and also by its close association and control of macrophage infiltration (Okunuki, Mukai et al.

464 2018). However, their appearance in our Rho-/-model indicate that these mechanisms may be

465 disadvantageous under different conditions. Activation of LPL on reaching the SRS may give

466 microglia an enhanced phagocytic capacity which may cause premature phagocytosis of

467 otherwise functional photoreceptor cells/segments. Phagocytosis of damaged and dying

468 photoreceptors is important to protect neighbouring healthy cells but overt phagocytosis may

469 enable retinal degeneration to advance at a more progressive rate than otherwise possible.

470 As microglia participate in vital physiological functions in the retina, complete

471 obliteration or blocking of all retinal microglial functions is undesirable (Wang, Zhao et al.

472 2016). Therefore, immunotherapeutic approaches that focus on targeting specific aspects of the

473 microglial phagocytic pathway, e.g. LPL, TYROBP, may be of benefit. Alternatively, targeting

474 the lipid catabolism pathway directly may provide another therapeutic avenue. Furthermore,

475 dampening these over-reactive microglia with minocycline in combination with drugs that target

476 photoreceptor cell death, or promote cell survival pathways, may present a broadly applicable

477 therapy more readily accessible for people with inherited retinopathies.

479 Conclusions: Overall, our study provides compelling evidence that targeting excessive microglia 480 phagocytosis may be a druggable intervention for some retinal degenerative diseases, and 481 identifies microglial lipid processing enzyme and DAM-marker LPL as a direct target of 482 minocycline indicating that suppression of lipid metabolism is one mechanism by which 483 minocycline protects against inflammation induced photoreceptor cell death. 


\section{Figure Legends}

487 Figure 1: Microglia are activated and infiltrate the outer retina over time in the rhodopsin 488 knockout $\left(\boldsymbol{r h o}^{-/-}\right)$model of photoreceptor degeneration.

489 (A) Retinal flatmounts from WT and Rho-/-mice at 3, 6, 9 and 12 weeks of age stained with the 490 microglia marker Ibal (20X magnification) with (a'-h') magnified and skeleton images. (B)

491 Retinal cryosections from WT and Rho-/-mice at 3, 6, 9 and 12 weeks of age stained with the 492 microglia marker Ibal (40X magnification, nuclei staining - Hoechst), with (a'-h') magnified 493 images of the ONL and sub-retinal space shown to the right. (IPL - inner plexiform layer, INL 494 inner nuclear layer, ONL outer nuclear layer).

Figure 2: Microglia infiltrating the sub-retinal space are actively phagocytosing 497 photoreceptor cells

498 (A) Retinal cryosections from WT mice and Rho-/-mice at 3, 6, 9 and 12 weeks of age stained 499 with Iba1 and CD68 (40X magnification) with magnified images of Rho-/-mice at (a') 3 and (b') 5006 weeks. (B) Retinal cryosections from WT mice and Rho-/-mice at 3, 6, 9 and 12 weeks of age 501 stained with P2RY12 and CD68 (40X magnification) with magnified images of Rho-/-mice at 502 (a') 3 and (b') 6 weeks (nuclei staining - Hoechst, arrows indicate areas of co-staining). (IPL 503 inner plexiform layer, INL - inner nuclear layer, ONL outer nuclear layer, LPL - lipoprotein 504 lipase).

505

506 Figure 3: Microglia infiltrating the sub-retinal space express disease-associated microglia 507 (DAM) marker LPL. 
508 (A) Retinal cryosections from WT mice and Rho-/-mice at 3, 6, 9 and 12 weeks of age stained 509 with Iba1 and LPL (40X magnification) with magnified images of Rho-/-mice at (a') 3, (b') 6, 510 (c') 9 and (d') 12 weeks (nuclei staining - Hoechst, arrows indicate areas of co-staining). (IPL 511 inner plexiform layer, INL - inner nuclear layer, ONL outer nuclear layer, LPL - lipoprotein 512 lipase).

514 Figure 4: Minocycline regulates microglia activation, downregulating DAM genes in the 515 rhodopsin knockout $\left(\right.$ rho $\left.^{-/}\right)$model of retinal degeneration.

516 (A) Ibal-stained retinal flatmounts from Rho-/-mice at 3 weeks of age given intra-peritoneal 517 injections of vehicle or minocycline $(50 \mathrm{mg} / \mathrm{kg})$ twice daily from post-natal day 16 (P16) to P21 518 with (B) microglial activation quantified by area grid analysis. (C) RT-PCR analysis of 519 homeostatic and DAM gene transcript levels in the neural retina from these minocycline- or 520 vehicle-injected Rho-/-mice at 3 weeks of age with a (D) heatmap showing the level of 521 expression normalized to the housekeeping gene ubiquitin $\mathrm{C}(* P \leq 0.05, * * P \leq 0.01, * * * P \leq$ 5220.001 by $t$ test, $n=5-6$ mice). (E) Retinal cryosections from these minocycline- or vehicle523 injected Rho-/-mice at 3 weeks of age stained with Ibal and the DAM gene LPL. (IPL - inner 524 plexiform layer, INL - inner nuclear layer, ONL outer nuclear layer, LPL - lipoprotein lipase). Figure 5: Minocycline directly regulates DAM gene expression in BV2 microglia in vitro.

527 (A) RT-PCR analysis of homeostatic and DAM gene transcript levels in BV2 microglial cells 528 that were untreated or treated with $5 \mathrm{ug} / \mathrm{ml}$ Minocycline for $7 \mathrm{~h}$ with a (B) heatmap showing the 529 level of expression normalized to the housekeeping gene ubiquitin $\mathrm{C}(* * * P \leq 0.001$ by $t$ test, $n=$ 5303 biological replicates). (C, D, E, F) Western blot analysis of LPL, APOE, CSF1R and Galectin- 
5313 expression levels in BV2 microglial cells that were untreated or treated with $5 \mathrm{ug} / \mathrm{ml}$ 532 minocycline for $24 \mathrm{~h}$ with densitometry shown to the right $(* * P \leq 0.01, * * * P \leq 0.001$ by $t$ test, $n$ $533=3$ biological replicates). survival in the rhodopsin knockout $\left(\right.$ rho $\left.^{-/}\right)$model of retinal degeneration.

537 (A, B, C, D) Iba1- and CD68-stained retinal cryosections from Rho-/-mice at 3 weeks and 6 538 weeks of age given intra-peritoneal injections of vehicle or minocycline $(50 \mathrm{mg} / \mathrm{kg})$ twice daily 539 from post-natal day $16(\mathrm{P} 16)$ to $\mathrm{P} 21$ or from P16 to P42 with microglial activation quantified by 540 area grid analysis. (E) Immunocytochemistry of BV2 microglial cells that were untreated or 541 treated with $5 \mathrm{ug} / \mathrm{ml}$ minocycline for $24 \mathrm{~h}$ and exposed to fluorescent opsonized latex beads for $5423 \mathrm{~h}$ before fixation and stained with MitoTracker and Hoechst. (F) Quantification of BV2 543 phagocytic activity expressed as percentage of bead ${ }^{+}$cells $(* * * P \leq 0.001$ by $t$ test, data 544 representative of 3 separate experiments with 3 fields of view imaged per well). (G, H) Optical 545 coherence tomography (OCT) images taken in vivo from from Rho-/-mice at 6 weeks of age 546 given intra-peritoneal injections of vehicle or minocycline $(50 \mathrm{mg} / \mathrm{kg})$ twice daily from post547 natal P16 to P42 with quantification of the ONL to the outer segment (OS) width (marked with 548 red line in the OCT images) using ImageJ ( $* P \leq 0.05, n=3-6$ mice per group). (I, J, K) 549 Haemotoxylin and eosin staining of paraffin-embedded sections from these minocycline- or 550 vehicle-treated Rho-/-mice at 6 weeks with quantification of the number of photoreceptor rows in 551 the ONL and quantification of the inner and outer segment length using ImageJ $(* P \leq 0.05, n=$ 552 3-6 mice per group). (GCL - ganglion cell layer, INL - inner nuclear layer, ONL outer nuclear 553 layer). 


\section{Abbreviations:}

555 Apolipoprotein E (APOE)

556 central nervous system (CNS)

557 colony stimulating factor 1 (CSF1R)

558 disease associated microglia (DAM)

559 external limiting membrane (ELM)

560 Haemotoxylin and Eosin (H\&E)

561 inner plexiform (IPL)

562 Ionized calcium binding adaptor molecule 1 (Iba1)

563 Leber congenital amaurosis (LCA)

564 lipoprotein lipase (LPL)

565 Optical coherence tomography (OCT)

566 outer nuclear layer (ONL)

567 outer plexiform layers (OPL)

568 Phosphate buffer saline (PBS)

569 Retinitis pigmentosa (RP)

570 rhodopsin knockout mice $\left(r h o^{-/-}\right)$

571 Ribonucleic acid (RNA)

572 sub-retinal space (SRS)

573 Transmembrane Immune Signaling Adaptor (TYROBP)

574 Triggering Receptor Expressed On Myeloid Cells 2 (TREM2)

575 Purinergic Receptor P2Y12 (P2ry12)

576 


\section{Declarations}

578 Ethical approval: All animal studies carried out in the Smurfit Institute of Genetics in TCD

579 adhere to the principles laid out by the internal ethics committee at TCD, and all relevant

580 national licenses were obtained before commencement of all studies.

581 Consent for publication: Not applicable.

582 Availability of data and materials: Data sharing not applicable to theis article a no datasets were 583 generated during this current study.

584 Competing Interests: The authors declare that they have no competing interests

585 Funding: SFI-15 CDA/3497, SFI-18/TIDA/6067, IRCLA/2017/295,

586 HRB/MRCG-2018-08, NCRC/18/10, ERC (Retina Rhythm - 864522).

587 Authors contributions: EO and CD performed experiments and analysed data, SD directed the 588 research. EO, MC and SD wrote the manuscript. All authors read and approved the final

589 manuscript

590 Acknowledgements: Not applicable 
594 Audrain, M., J. V. Haure-Mirande, J. Mleczko, M. Wang, J. K. Griffin, P. H. St George-Hyslop, 595 P. Fraser, B. Zhang, S. Gandy and M. E. Ehrlich (2021). "Reactive or transgenic increase in 596 microglial TYROBP reveals a TREM2-independent TYROBP-APOE link in wild-type and 597 Alzheimer's-related mice." Alzheimers Dement 17(2): 149-163.

598 Dannhausen, K., C. Möhle and T. Langmann (2018). "Immunomodulation with minocycline 599 rescues retinal degeneration in juvenile neuronal ceroid lipofuscinosis mice highly susceptible to 600 light damage." Dis Model Mech 11(9).

601 Humphries, M. M., D. Rancourt, G. J. Farrar, P. Kenna, M. Hazel, R. A. Bush, P. A. Sieving, D. 602 M. Sheils, N. McNally, P. Creighton, A. Erven, A. Boros, K. Gulya, M. R. Capecchi and P. 603 Humphries (1997). "Retinopathy induced in mice by targeted disruption of the rhodopsin gene." $604 \quad$ Nat Genet 15(2): 216-219.

605 Karperien, A., H. Ahammer and H. F. Jelinek (2013). "Quantitating the subtleties of microglial 606 morphology with fractal analysis." Front Cell Neurosci 7: 3.

607 Keren-Shaul, H., A. Spinrad, A. Weiner, O. Matcovitch-Natan, R. Dvir-Szternfeld, T. K. Ulland, 608 E. David, K. Baruch, D. Lara-Astaiso, B. Toth, S. Itzkovitz, M. Colonna, M. Schwartz and I. 609 Amit (2017). "A Unique Microglia Type Associated with Restricting Development of 610 Alzheimer's Disease." Cell 169(7): 1276-1290.e1217.

611 Kobayashi, K., S. Imagama, T. Ohgomori, K. Hirano, K. Uchimura, K. Sakamoto, A. Hirakawa, 612 H. Takeuchi, A. Suzumura, N. Ishiguro and K. Kadomatsu (2013). "Minocycline selectively 613 inhibits M1 polarization of microglia." Cell Death Dis 4(3): e525. 
614 Kohno, H., Y. Chen, B. M. Kevany, E. Pearlman, M. Miyagi, T. Maeda, K. Palczewski and A. 615 Maeda (2013). "Photoreceptor proteins initiate microglial activation via Toll-like receptor 4 in 616 retinal degeneration mediated by all-trans-retinal." J Biol Chem 288(21): 15326-15341.

617 Krasemann, S., C. Madore, R. Cialic, C. Baufeld, N. Calcagno, R. El Fatimy, L. Beckers, E. 618 O'Loughlin, Y. Xu, Z. Fanek, D. J. Greco, S. T. Smith, G. Tweet, Z. Humulock, T. Zrzavy, P. 619 Conde-Sanroman, M. Gacias, Z. Weng, H. Chen, E. Tjon, F. Mazaheri, K. Hartmann, A. Madi, J. 620 D. Ulrich, M. Glatzel, A. Worthmann, J. Heeren, B. Budnik, C. Lemere, T. Ikezu, F. L. Heppner, 621 V. Litvak, D. M. Holtzman, H. Lassmann, H. L. Weiner, J. Ochando, C. Haass and O. Butovsky 622 (2017). "The TREM2-APOE Pathway Drives the Transcriptional Phenotype of Dysfunctional 623 Microglia in Neurodegenerative Diseases." Immunity 47(3): 566-581.e569.

624 Loving, B. A. and K. D. Bruce (2020). "Lipid and Lipoprotein Metabolism in Microglia." Front $625 \quad$ Physiol 11: 393.

626 Ma, J., C. Becker, C. A. Lowell and D. M. Underhill (2012). "Dectin-1-triggered recruitment of 627 light chain 3 protein to phagosomes facilitates major histocompatibility complex class II 628 presentation of fungal-derived antigens." J Biol Chem 287(41): 34149-34156.

629 Ma, J., T. Jiang, L. Tan and J. T. Yu (2015). "TYROBP in Alzheimer's disease." Mol Neurobiol $630 \quad 51(2): 820-826$.

631 Ma, Y., J. Bao, X. Zhao, H. Shen, J. Lv, S. Ma, X. Zhang, Z. Li, S. Wang, Q. Wang and J. Ji 632 (2013). "Activated cyclin-dependent kinase 5 promotes microglial phagocytosis of fibrillar $\beta$ 633 amyloid by up-regulating lipoprotein lipase expression." Mol Cell Proteomics 12(10): 28336342844.

635 Murakami, Y., K. Ishikawa, S. Nakao and K. H. Sonoda (2020). "Innate immune response in 636 retinal homeostasis and inflammatory disorders." Prog Retin Eye Res 74: 100778. 
637 O'Koren, E. G., C. Yu, M. Klingeborn, A. Y. W. Wong, C. L. Prigge, R. Mathew, J. Kalnitsky, 638 R. A. Msallam, A. Silvin, J. N. Kay, C. Bowes Rickman, V. Y. Arshavsky, F. Ginhoux, M. 639 Merad and D. R. Saban (2019). "Microglial Function Is Distinct in Different Anatomical 640 Locations during Retinal Homeostasis and Degeneration." Immunity 50(3): 723-737.e727.

641 Okunuki, Y., R. Mukai, E. A. Pearsall, G. Klokman, D. Husain, D. H. Park, E. Korobkina, H. L. 642 Weiner, O. Butovsky, B. R. Ksander, J. W. Miller and K. M. Connor (2018). "Microglia inhibit 643 photoreceptor cell death and regulate immune cell infiltration in response to retinal detachment." $644 \quad$ Proc Natl Acad Sci U S A 115(27): E6264-e6273.

645 Ozaki, E., L. Gibbons, N. G. Neto, P. Kenna, M. Carty, M. Humphries, P. Humphries, M. 646 Campbell, M. Monaghan, A. Bowie and S. L. Doyle (2020). "SARM1 deficiency promotes rod 647 and cone photoreceptor cell survival in a model of retinal degeneration." Life Sci Alliance 3(5).

648 Peng, B., J. Xiao, K. Wang, K. F. So, G. L. Tipoe and B. Lin (2014). "Suppression of microglial 649 activation is neuroprotective in a mouse model of human retinitis pigmentosa." $\underline{\mathrm{J} \text { Neurosci }}$ $650 \quad 34(24): 8139-8150$.

651 Scholz, R., M. Sobotka, A. Caramoy, T. Stempfl, C. Moehle and T. Langmann (2015). 652 "Minocycline counter-regulates pro-inflammatory microglia responses in the retina and protects 653 from degeneration." J Neuroinflammation 12: 209.

654 Terauchi, R., H. Kohno, S. Watanabe, S. Saito, A. Watanabe and T. Nakano (2021). 655 "Minocycline decreases CCR2-positive monocytes in the retina and ameliorates photoreceptor 656 degeneration in a mouse model of retinitis pigmentosa." PLoS One 16(4): e0239108.

657 Wang, X., L. Zhao, J. Zhang, R. N. Fariss, W. Ma, F. Kretschmer, M. Wang, H. H. Qian, T. C. 658 Badea, J. S. Diamond, W. B. Gan, J. E. Roger and W. T. Wong (2016). "Requirement for 
659 Microglia for the Maintenance of Synaptic Function and Integrity in the Mature Retina." $\underline{\mathrm{J}}$ 660 Neurosci 36(9): 2827-2842.

661 Zhang, C., B. Lei, T. T. Lam, F. Yang, D. Sinha and M. O. Tso (2004). "Neuroprotection of 662 photoreceptors by minocycline in light-induced retinal degeneration." Invest Ophthalmol Vis Sci 663 45(8): 2753-2759.

664 
Figure 1

A
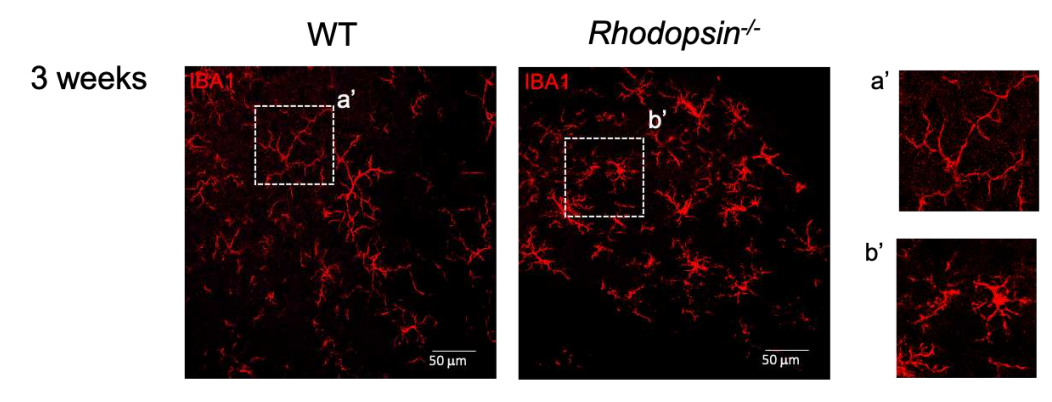

WT
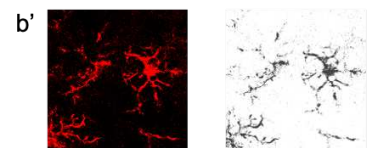

Rho--

6 weeks
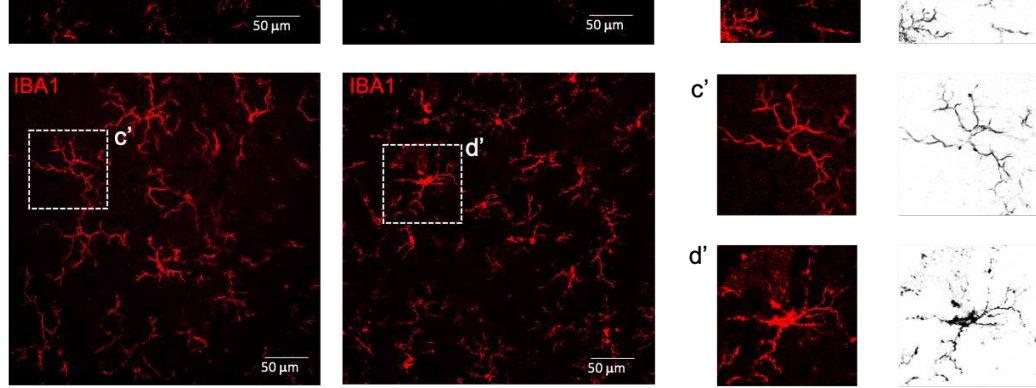

R.

d'

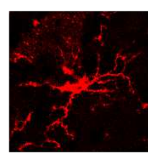

$+2$

Rho-

9 weeks
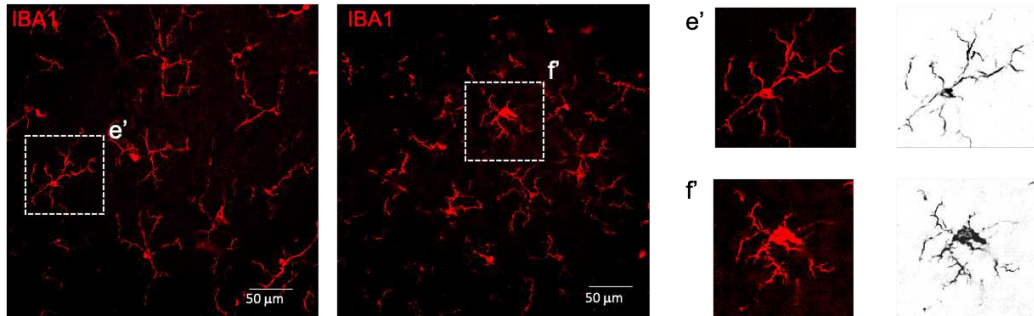

WT
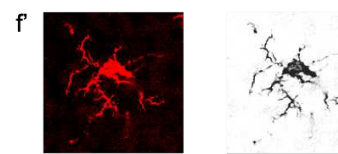

Rho'

12 weeks
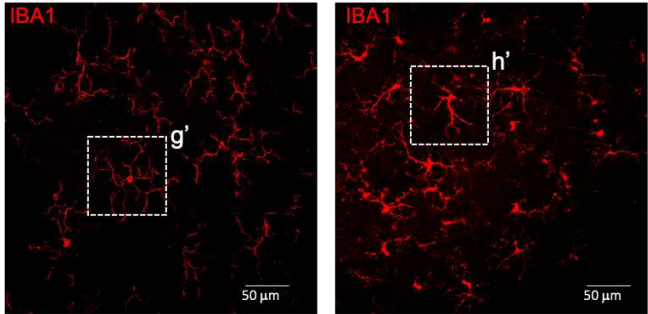

g'
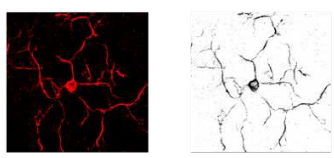

WT

h

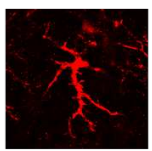

R $\quad$ Rho

Rhodopsin-

3 weeks
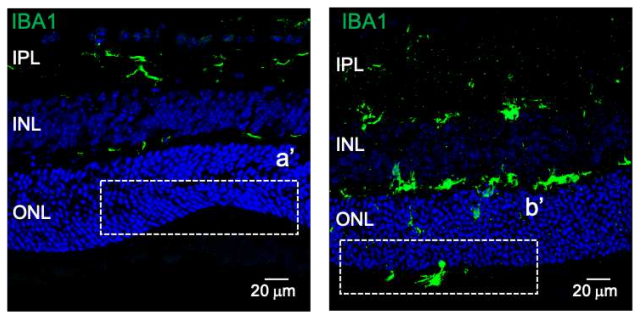

3 weeks

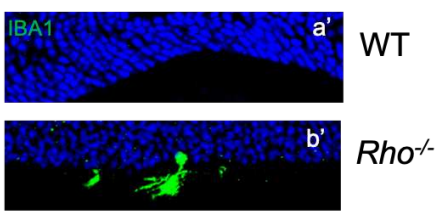

6 weeks
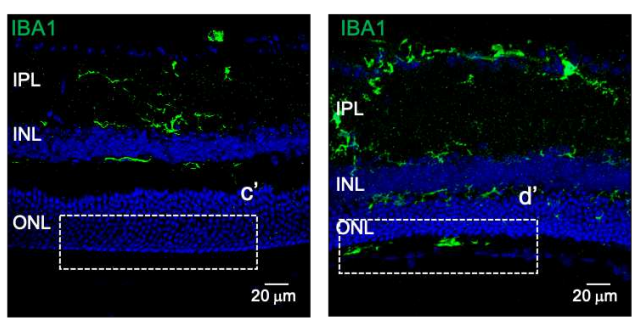

6 weeks
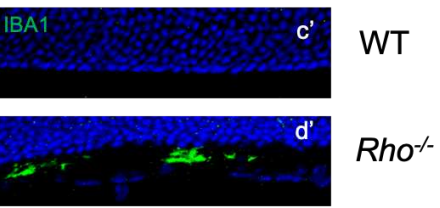

9 weeks
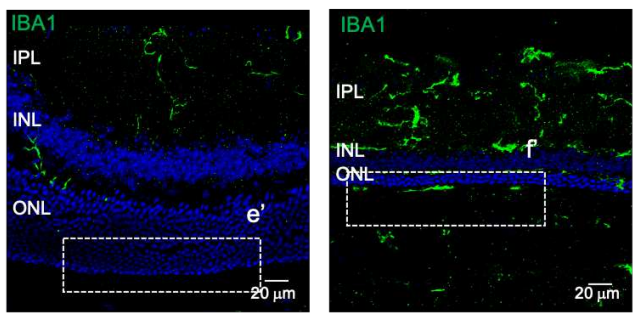

9 weeks
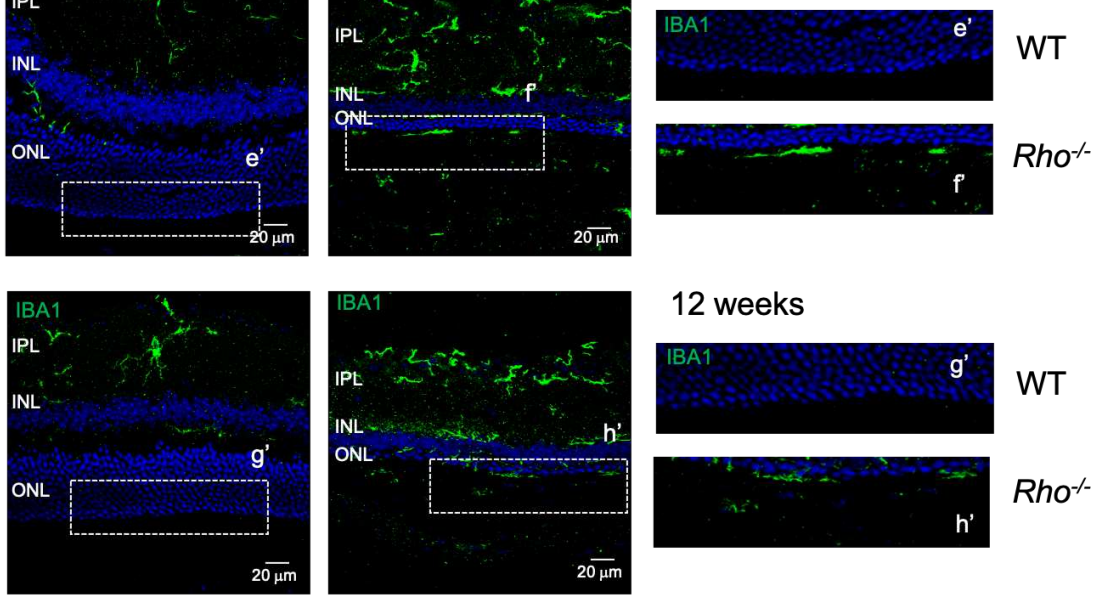

12 weeks

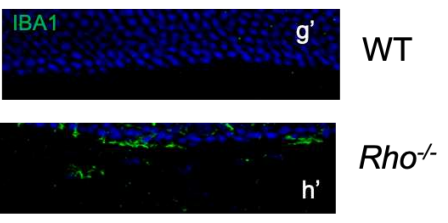


Figure 2

A
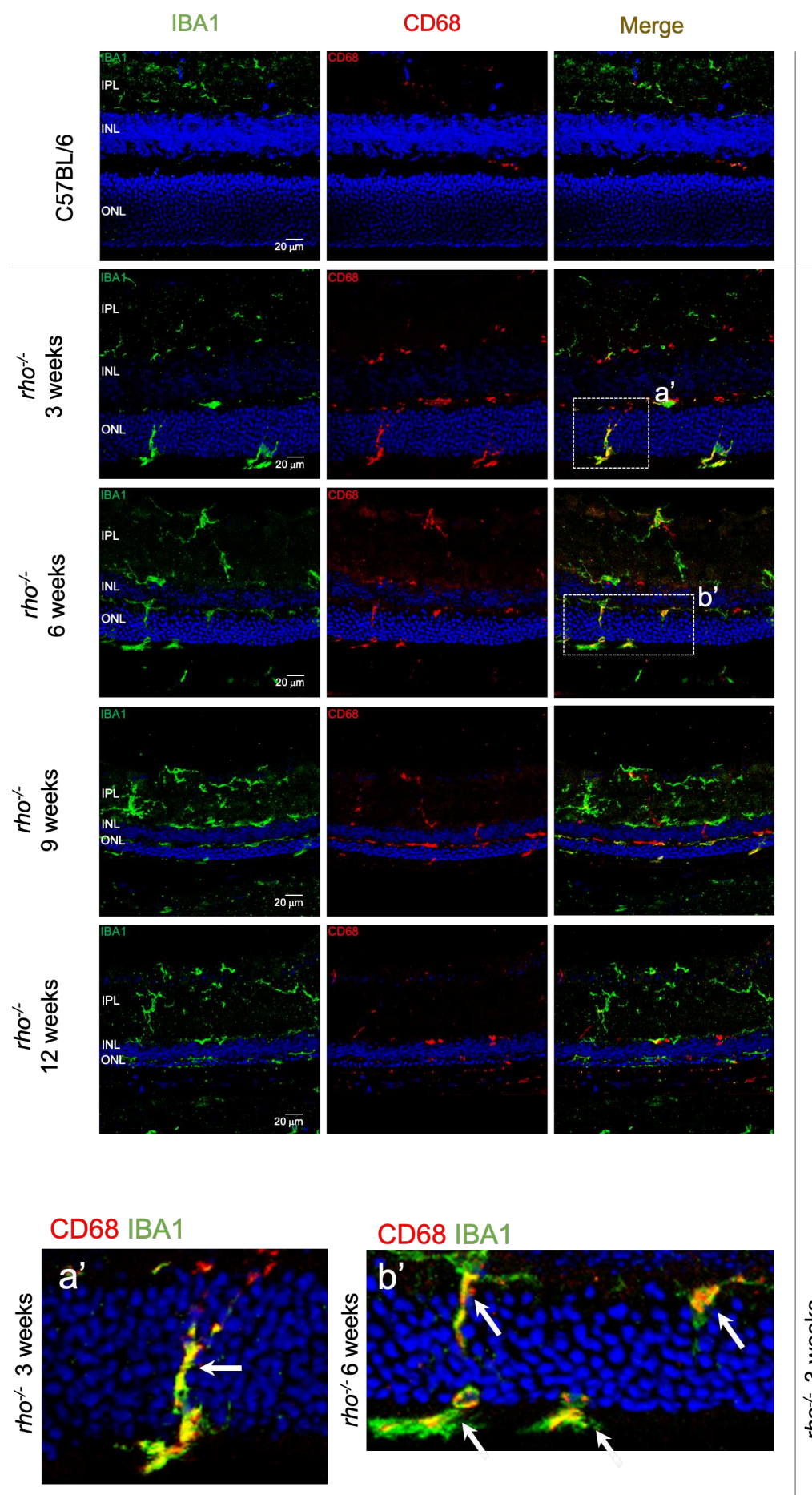

B

P2RY12

Merge
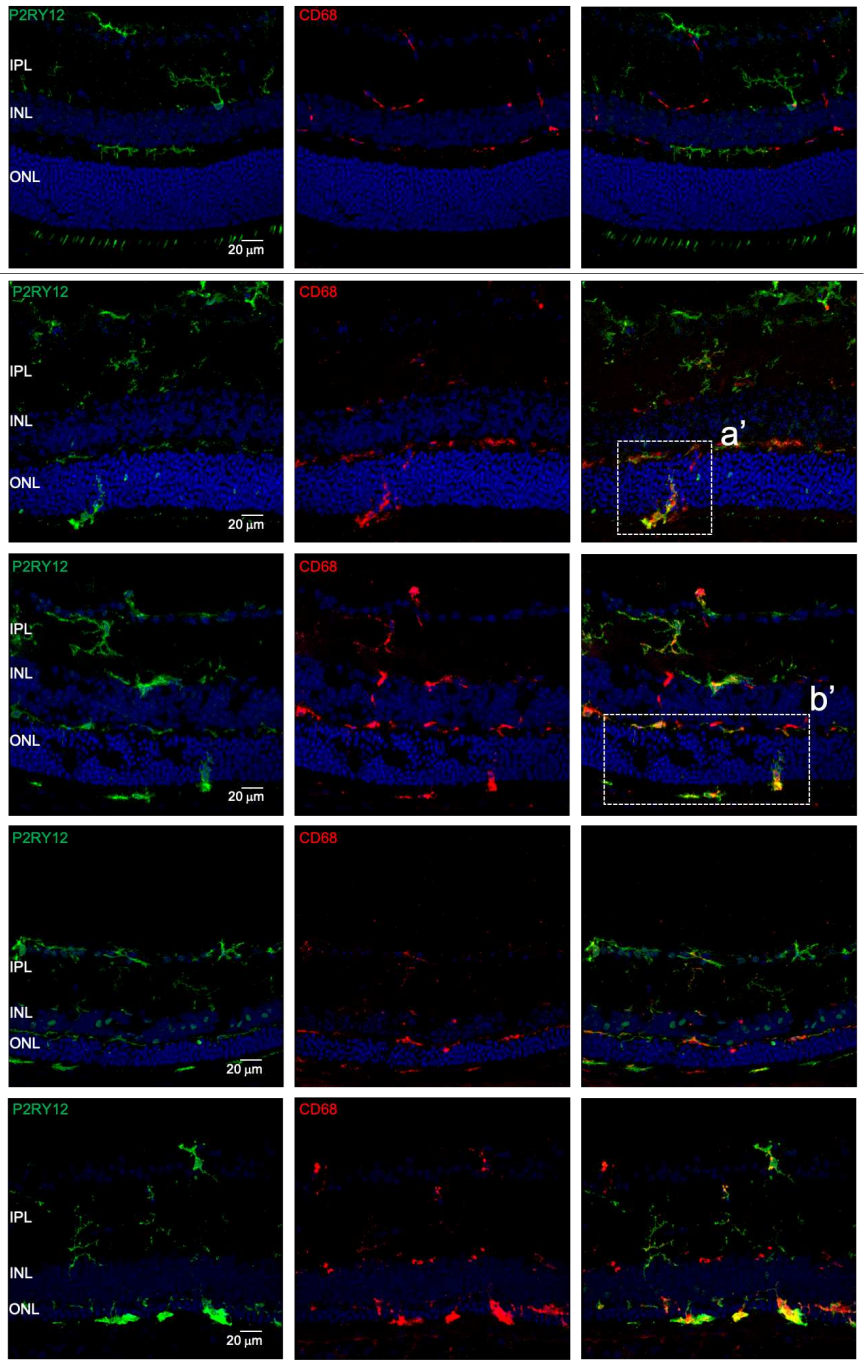

P2RY12 CD68

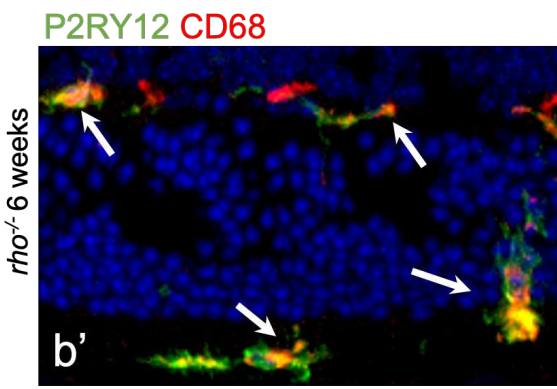


Figure 3
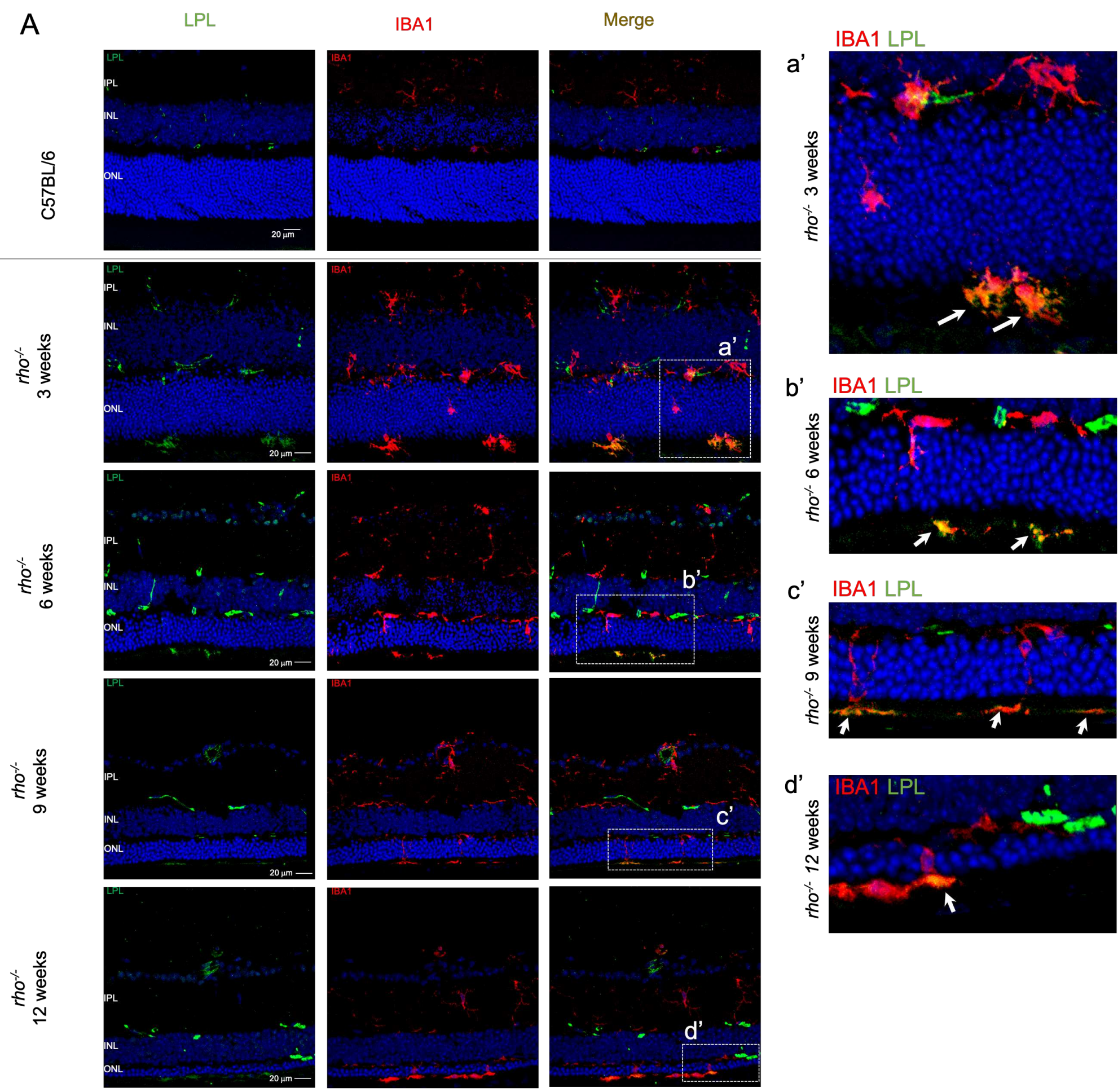

b' IBA1 LPL

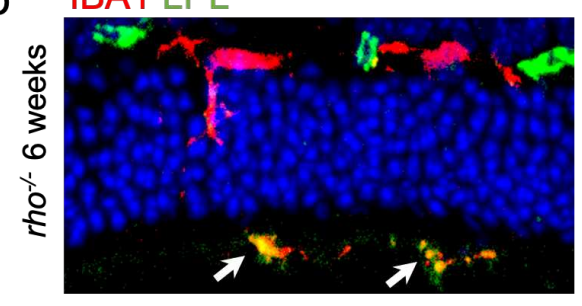

c' IBA1 LPL
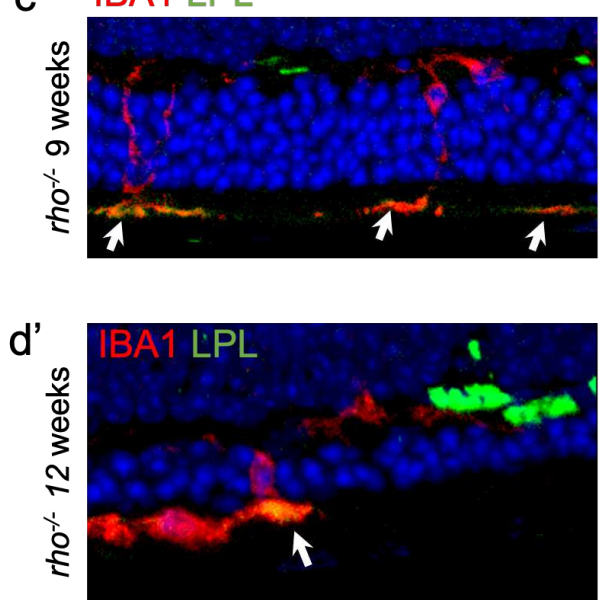
Figure 4
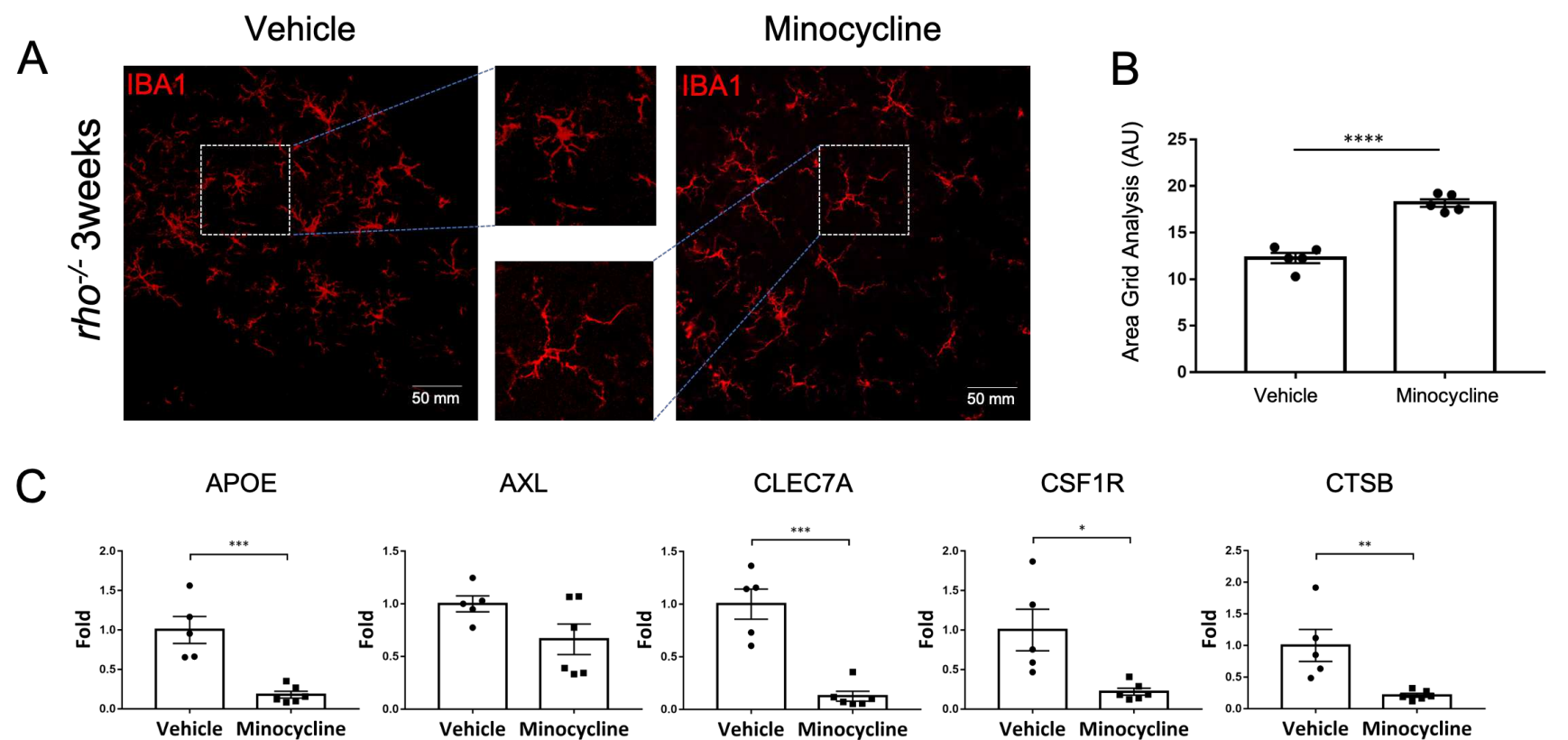

$\begin{array}{llll}\text { CX3CR1 ITGAX LGALS3 } & \text { LPL }\end{array}$
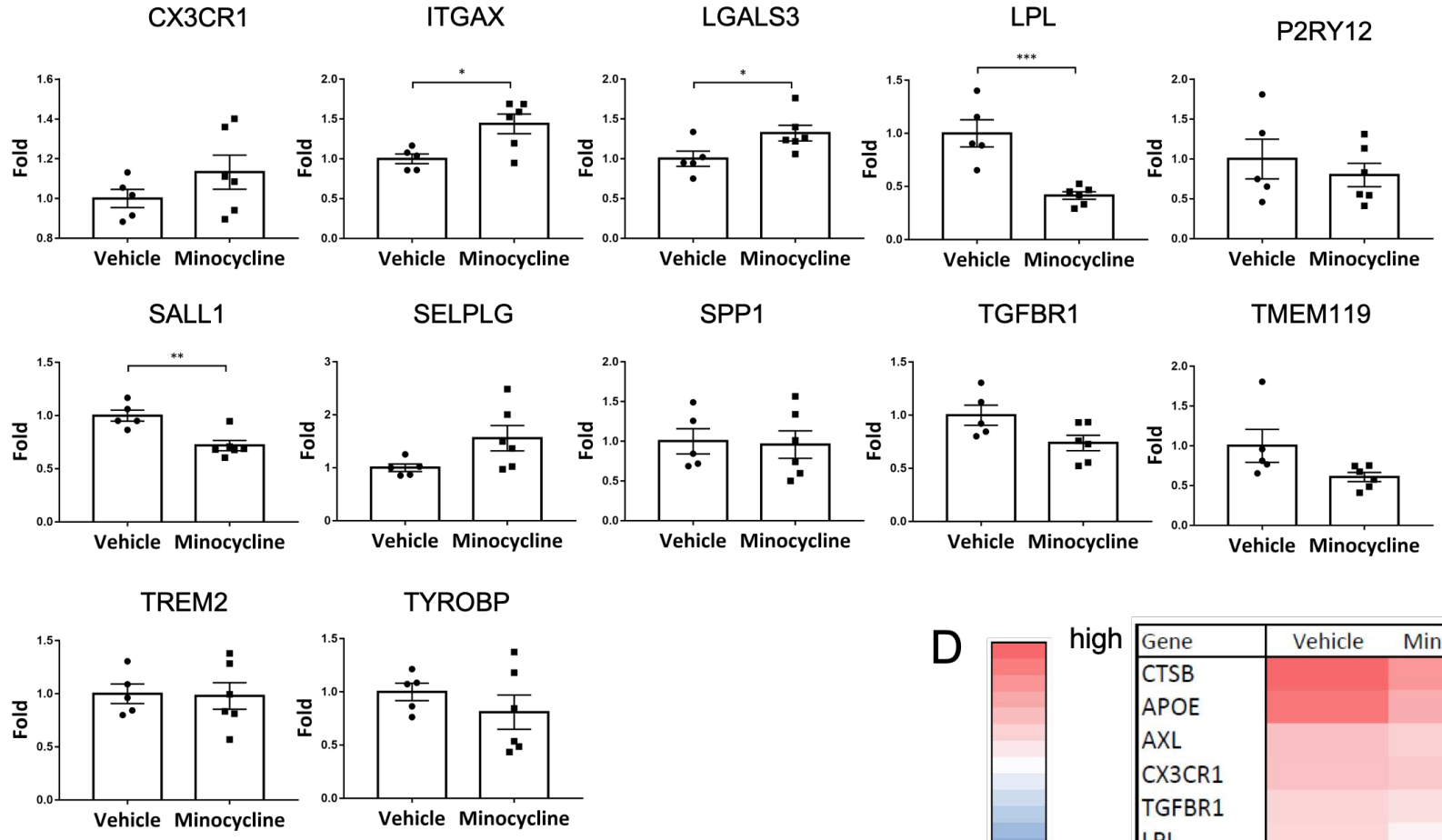

E

LPL

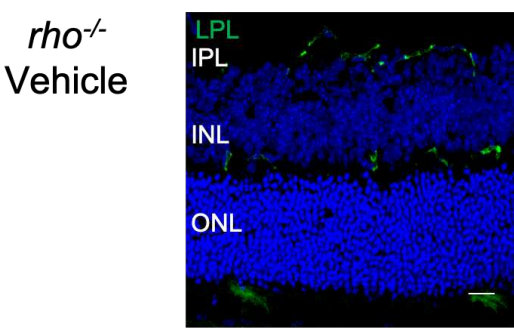

rho $^{-/} \quad \frac{\mathrm{LPL}}{\mathrm{IPL}}$

Minocycline INL
IBA1
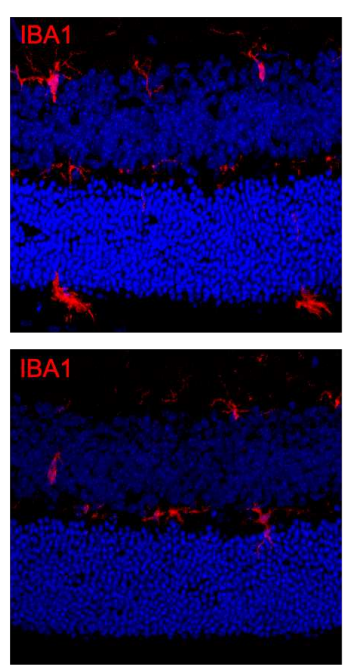

D

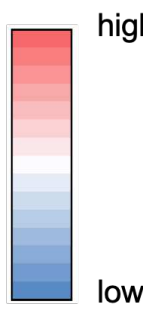

Merge
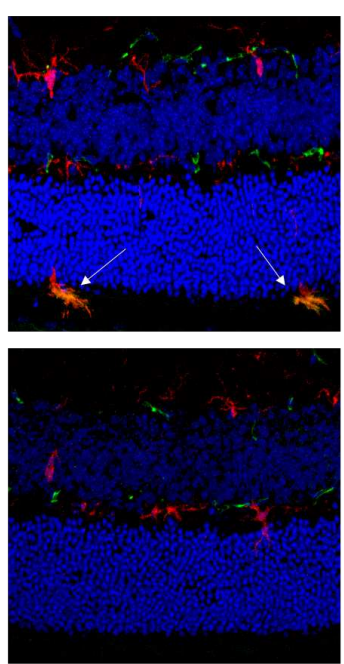
Figure 5

A

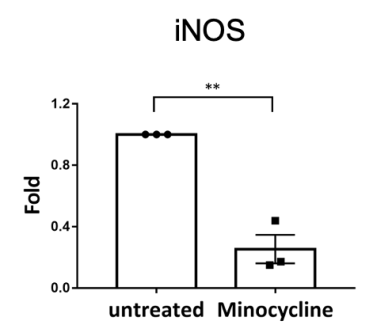

CSF1R

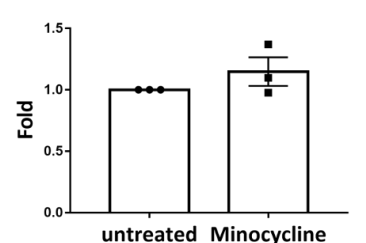

LGALS3
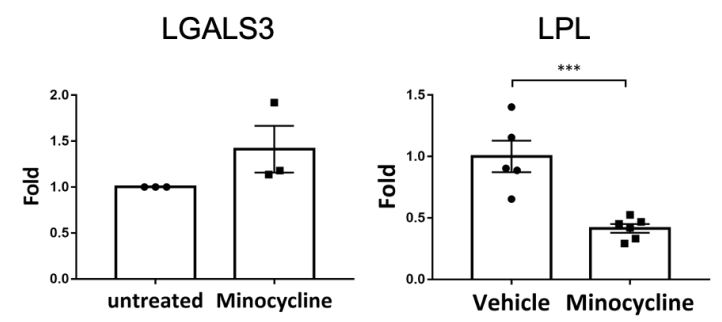

SELPLG
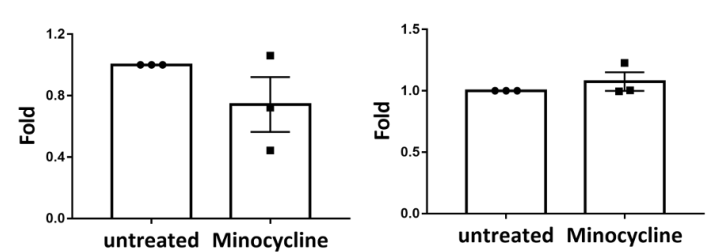

TREM2

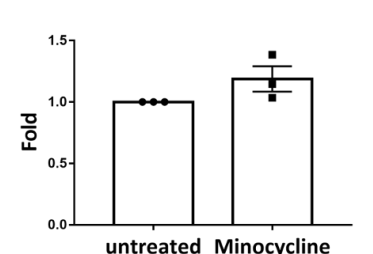

SPP1

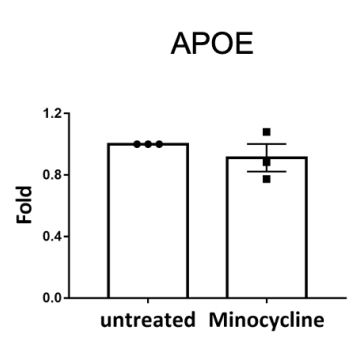

CTSB
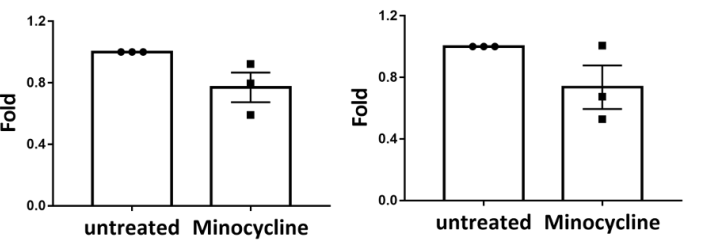

P2RY12

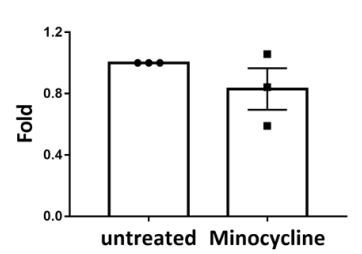

TGFBR1
AXL
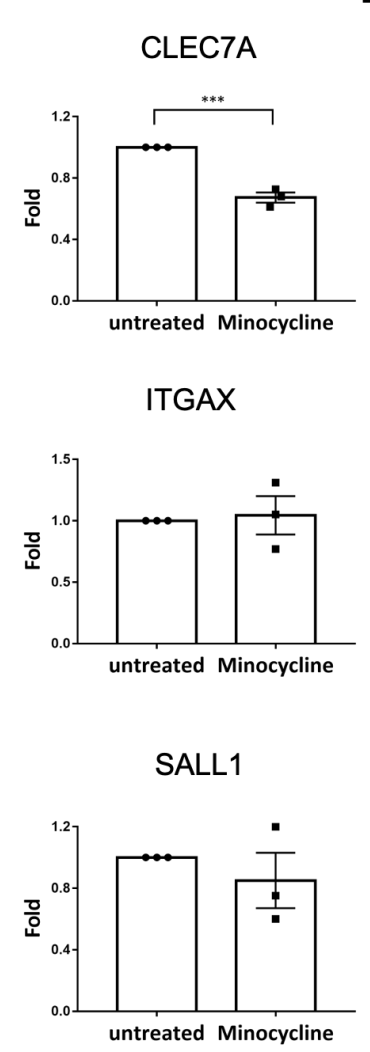

흔

TMEM119
B

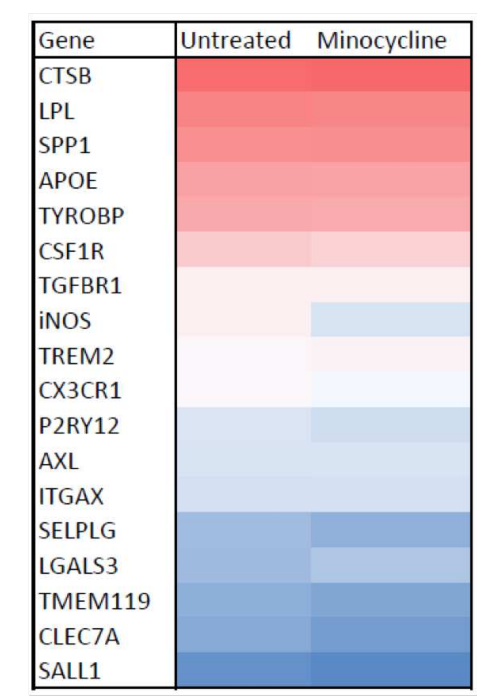

C
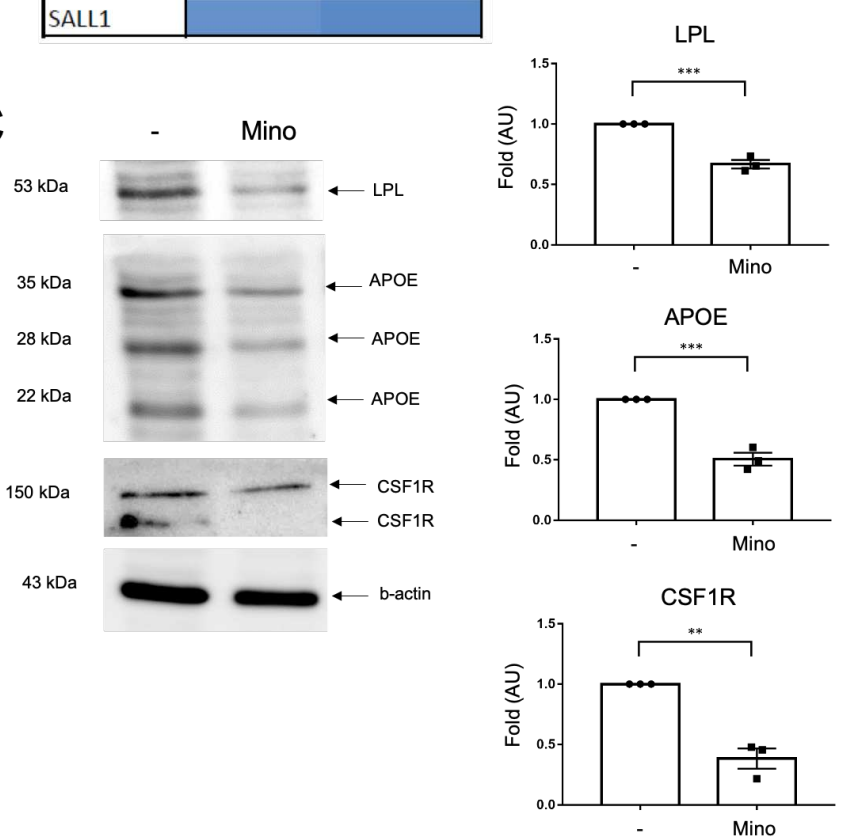

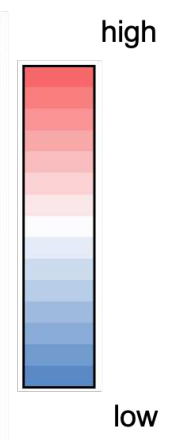

D

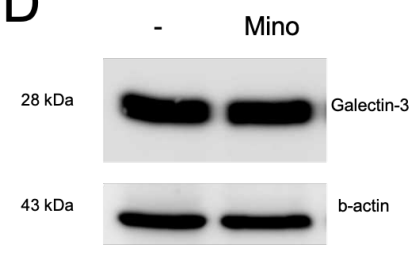

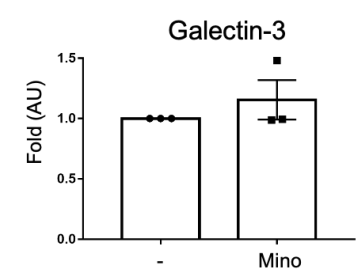


Figure 6
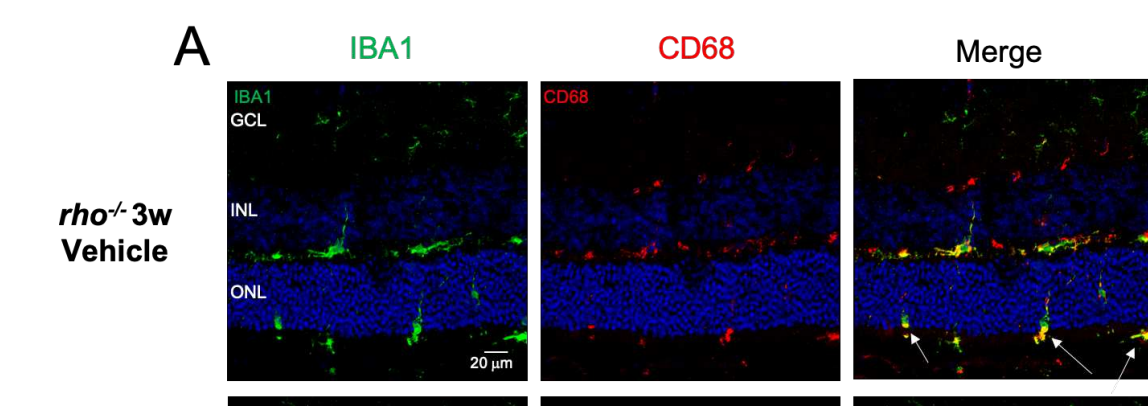

E
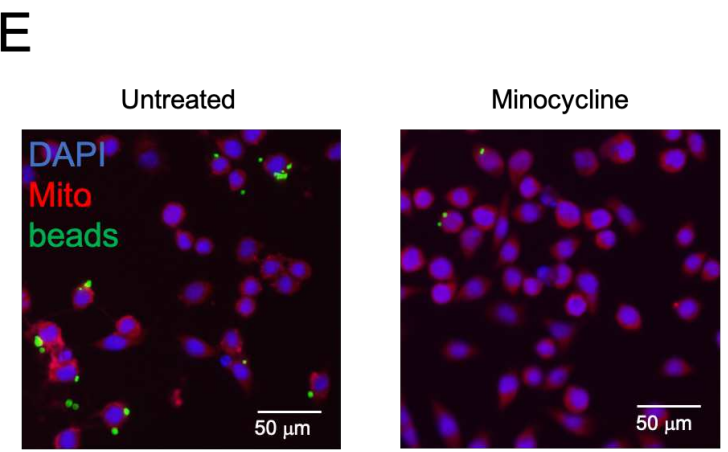

F

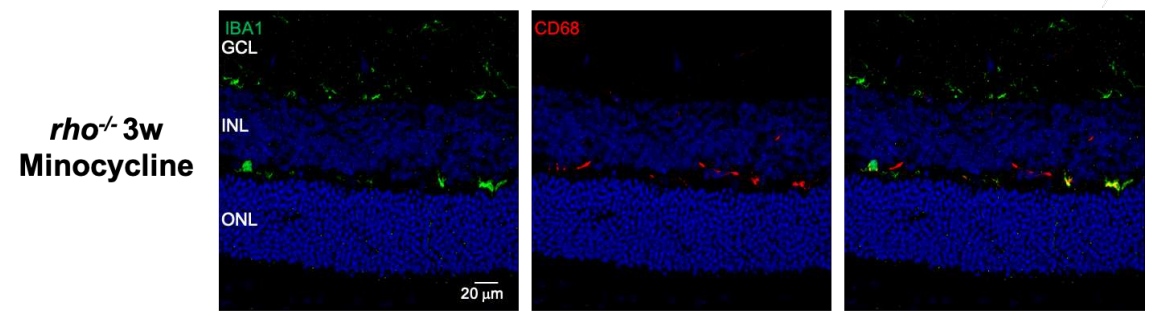

G

$\mathrm{H}$

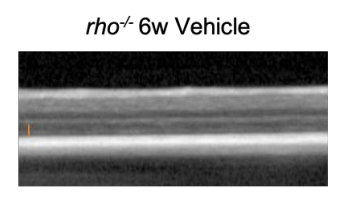

IBA1

CD68

Merge

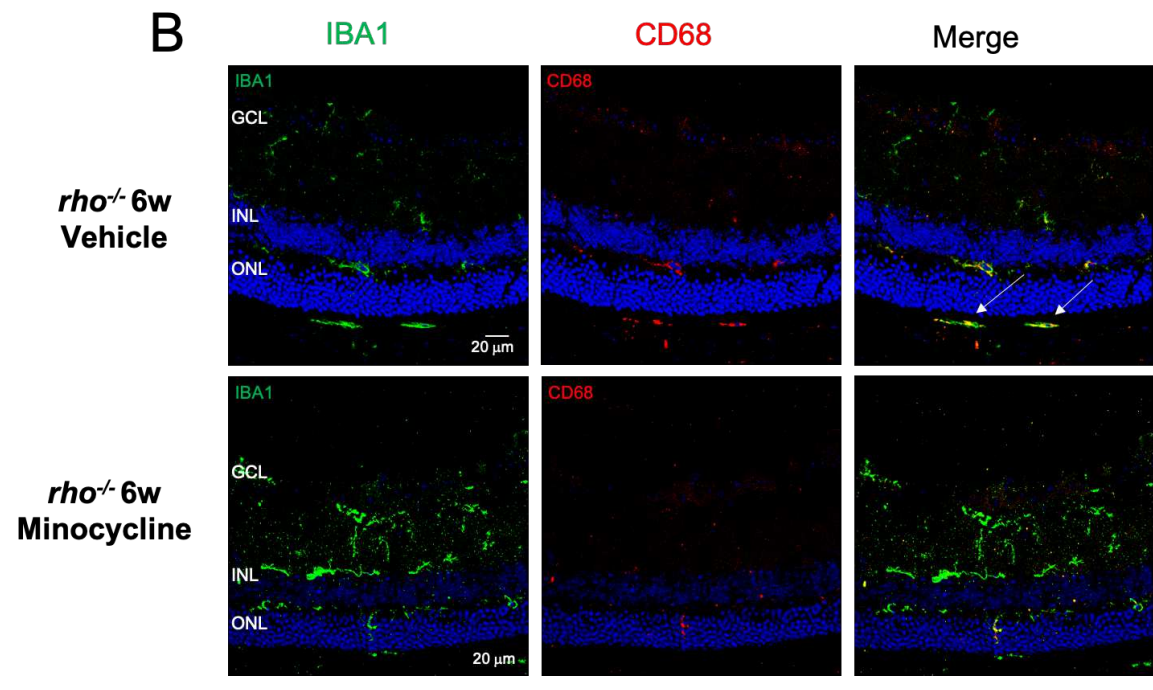

$r h 0^{-1-6 w}$ Minocycline
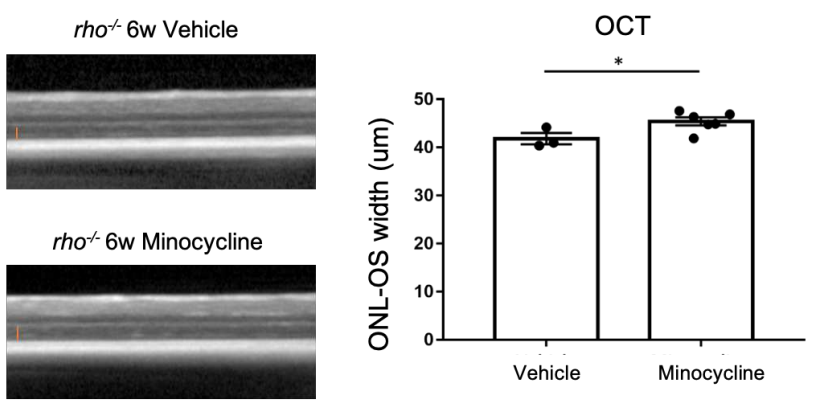

I

Vehicle
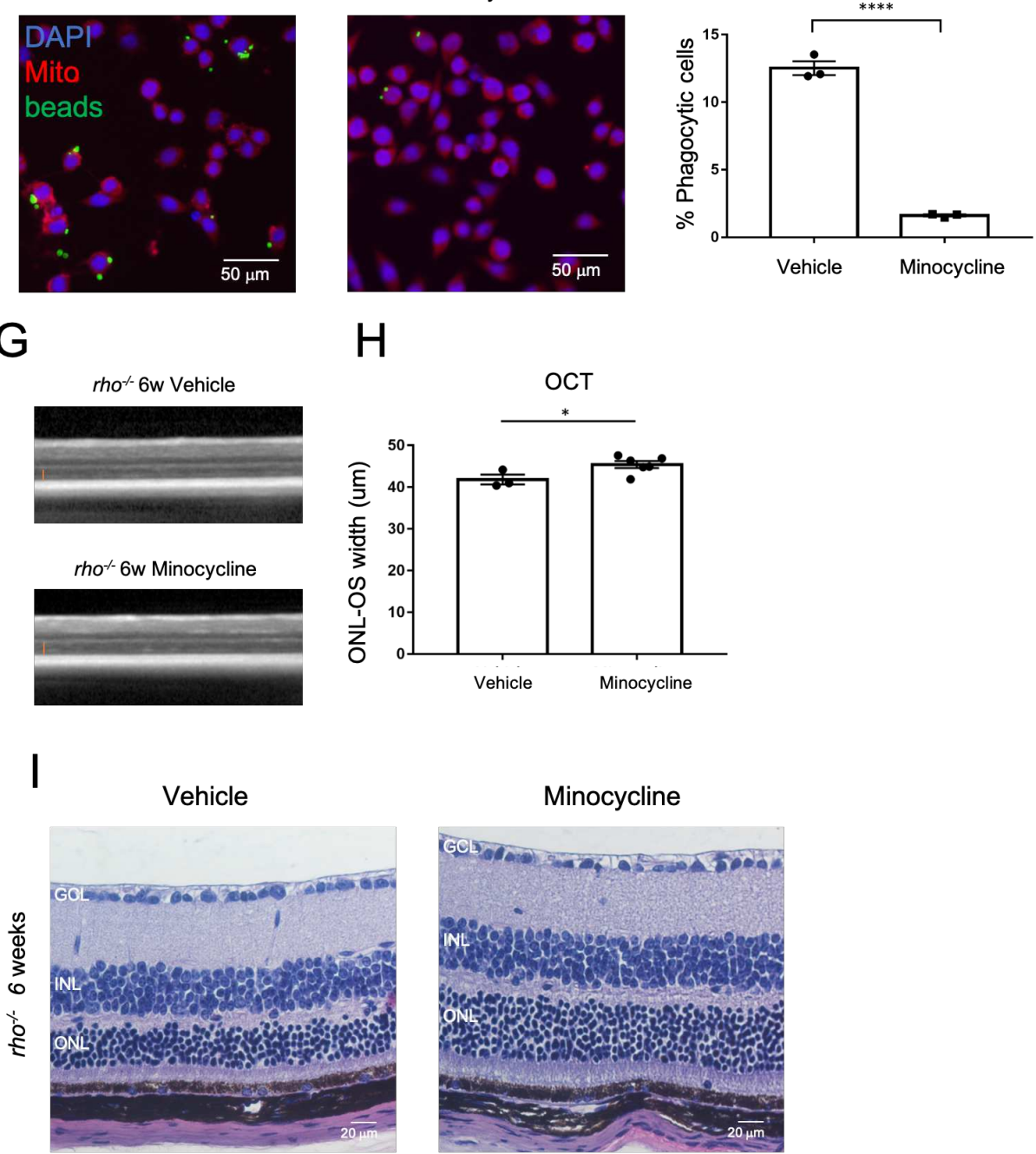

C

D
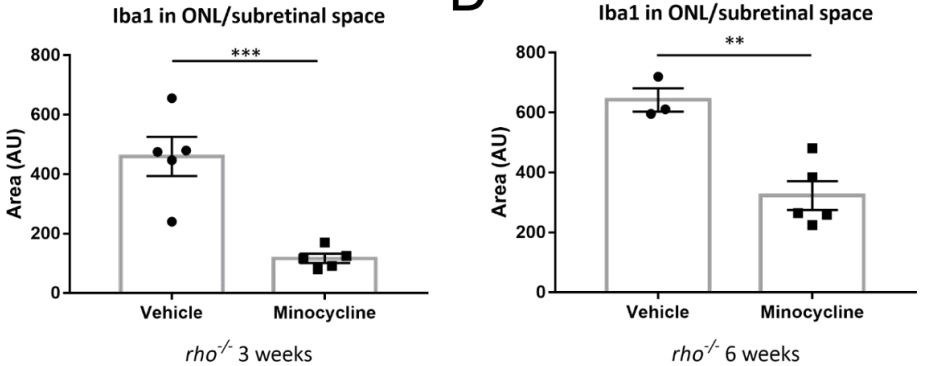

J

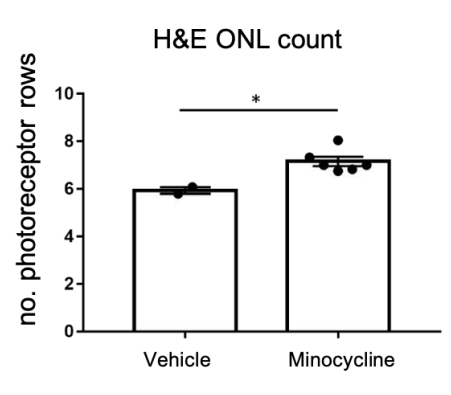

K
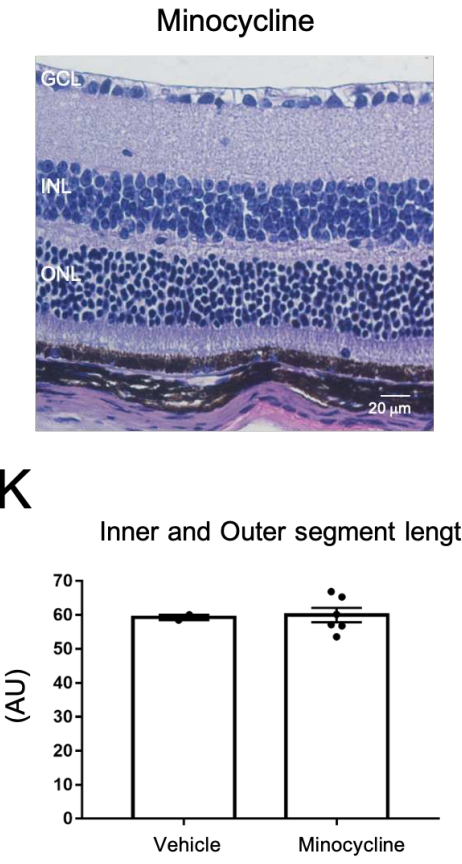